22. Hashim, N. L. S., Yahya, A., Andromeda, T., Kadir, M. R. R. A., Mahmud, N., Samion, S. (2012). Simulation of PSO-PI Controller of DC Motor in Micro--EDM System for Biomedical Application. Procedia Engineering, 41, 805-811. doi: https://doi.org/10.1016/ j.proeng.2012.07.247

23. Kennedy, J., Eberhart, R. (1995). Particle Swarm Optimization. Proceedings of ICNN'95 - International Conference on Neural Networks, 1942-1948. doi: https://doi.org/10.1109/icnn.1995.488968

24. Shi, Y., Eberhart, R. (1998). A modified particle swarm optimizer. 1998 IEEE International Conference on Evolutionary Computation Proceedings. IEEE World Congress on Computational Intelligence (Cat. No.98TH8360). doi: https://doi.org/ 10.1109/icec.1998.699146

25. Zhan, Z.-H., Zhang, J., Li, Y., Chung, H. S.-H. (2009). Adaptive Particle Swarm Optimization. IEEE Transactions on Systems, Man, and Cybernetics, Part B (Cybernetics), 39 (6), 1362-1381. doi: https://doi.org/10.1109/tsmcb.2009.2015956

This paper considers the construction of principles and the synthesis of a system of effective control over the processes of synchronization of generator sets (GSs) that form a part of the distributed MP-control systems for complex ship technical systems and complexes (STS and C). The tasks of synchronization have been set, the process and database models have been built, the system configurations have been defined. Based on the use of resultant functions, we have determined stages in solving the tasks of control over the frequency adjustment synchronization in a hierarchical sequence. The performance analysis of the STS and $C$ control elements has been carried out; the use of the integrated optimization criteria and dual management principles has been proposed. Practical techniques to manage the GS synchronization have been given. We have solved the problem of high-speed control over the frequency of synchronized objects based on the principles of adjustment. That has made it possible to determine in advance the moments of GS enabling under the deterministic and stochastic statement of the synchronization task. The results of the experimental study into the GS synchronization processes are given; the effectiveness of the proposed GS control has been proven. The principles underlying the construction of procedures to control the GS composition when using the methods of "rigid" and "flexible" thresholds have made it possible to define the optimization criteria and implement a control law that satisfied the condition for an extremum, which is an indicator of the feasibility of the set goal and takes into consideration the limitations of control influences. We managed to design a system in the class of adaptive control systems by the appropriate decomposition of the system's elements by splitting $a$ synchronization task into the task on performance and the task on control under the required conditions. The given examples of the processes where the synchronization failed while using standard synchronizer control algorithms, as well as processes of successful GS synchronization when applying the proposed synchronizer dual control algorithms, have confirmed the reliability of the main scientific results reported here

Keywords: technical operation, synchronization, quality, control system, mathematical modeling

\section{Received date 04.01.2021}

\section{Introduction}

The use of microprocessor (MP) equipment in the ship's electricity generating units implies the automation of control processes, as well as the processes of control and protection of ship technical systems and complexes (STS and C). An important and integrated part of each of these processes is the measurement of electric power parameters, which, for example, are necessary to enable effective automatic synchronization of generator sets (GSs). Namely: the magnitude and nature of load currents in all phases, the voltage, frequency of the current, the phase angle $\varphi$ of the shift between the current and voltage, power direction, and others.
UDC 656.612 .8

DOI: $10.15587 / 1729-4061.2021 .225517$

THE SYNTHESIS OF CONTROL SYSTEM TO SYNCHRONIZE SHIP GENERATOR ASSEMBLIES

\author{
V. Bu d a s h ko \\ Doctor of Technical Sciences, Associate Professor \\ Educational and Scientific Institute of \\ Automation and Electromechanics \\ E-mail: bvv@te.net.ua \\ V. Shevchenko \\ $\mathrm{PhD}$, Associate Professor \\ Training \& Certifying Center of Seafarers* \\ E-mail: vash4891@gmail.com \\ *National University "Odessa Maritime Academy» \\ Didrikhsona str., 8, Odessa, Ukraine, 65029
}

Copyright (C) 2021, V. Budashko, V. Shevchenko This is an open access article under the CC BY license (http://creativecommons.org/licenses/by/4.0)

It is known that aboard a modern sea vessel most of the measurement systems for electric power parameters are associated with the functioning of analog-digital converters (ADC), and, along with the issue of ensuring reliability and accuracy, there is a task to provide for the necessary speed of measurements. Resolving this issue requires finding new ways - based on a systematic approach with accounting for known digital measurement techniques. In a general form, the task to measure the electrical energy parameters in an $n$-phase system relates to determining the set of values $\left\{I_{1}, \ldots, I_{n}, U_{1}, \ldots, U_{n}, f, \varphi\right\}$ according to the selected performance criterion. In this case, $I_{1}, \ldots, I_{n}$ and $U_{1}, \ldots, U_{n}$ are the amplitude values of phase currents and linear voltages. 
For a three-phase system, the most common case of the $n$-phase system, the measurement of electrical energy parameters is carried out by converting the shapers of rectangular pulses of linear voltages and phase currents of the generator into discrete signals reflecting the half-periods of their sinusoids. Sequential, byte-wise analysis of sets of input signals states makes it possible to determine the law (algorithm) of ADC control, the input of which receives normalized amplitude values of full currents of phases and linear voltages of the generator.

When solving a task of the multidimensional measurement of electricity parameters (currents, power, voltage, etc.), for reasons of efficiency and, in accordance with the selected criteria of accuracy and speed, fundamentally suitable are ADC with an error of 0.05 to $1 \%$ at a maximum conversion frequency of $30 \mathrm{MHz}$.

Therefore, devising effective methods for finding the rational configurations of various STS and C and their control systems implies a trade-off between efficiency, reliability of energy supply, and the environmental friendliness of ship automated electric power plants (SAEPP).

We can conclude that the development and construction of new, as well as the improvement of existing, control systems for technological processes of various complex STS and C are impossible without defining and applying modern theoretical methods to synthesize effective control techniques. Therefore, one of the relevant goals in the development of STS and C is to choose a SAEPP composition that would provide for the uninterrupted power supply of the required quality, at the maximum possible efficiency in a wide range of dynamic loads on GSs. On the other hand, it is impossible to improve the efficiency of vessel operations in general under changing weather conditions without taking into consideration the restrictions regarding the maximum permissible harmful emissions imposed by environmental factors.

\section{Literature review and problem statement}

In ship electricity engineering, GS synchronization refers to the most complex and responsible control processes. There are fundamental works [1] and modern mathematical methods [2] covering the research and design of electric power systems with static, dynamic, and variable structures [3]. These and other works make it possible to calculate GS currents during the disruption of synchronization conditions and to set valid limits [4]. We note the progress in the development of methods of abstract and structural synthesis of automatic synchronization devices [5], in particular using the achievements of modern electronics and the synthesis of algorithmic support for programmed MP systems [6]. To summarize, in the best case, the authors, for example, of [7], give a description of how the nodes of some typical synchronizers or algorithms work in the most general form. There is a task to analyze the existing techniques for automating the process of ship GS synchronization [8], to determine and define mathematical models of basic converters [9], to investigate them from the point of view of practical implementation in MP control systems [10]. Of course, it is necessary to strive for the maximum use of the positive properties of modern hardware and software logic according to the criteria of reliability, accuracy, performance, etc. [11]. The tasks to optimize control over the synchronization process in SAEPP with SG installations [12] remain unresolved.

Fig. 1 shows an example of the diagrams explaining the essence of the existing issue related to the process of measuring electrical energy with the help of shapers of rectangular pulses [13]
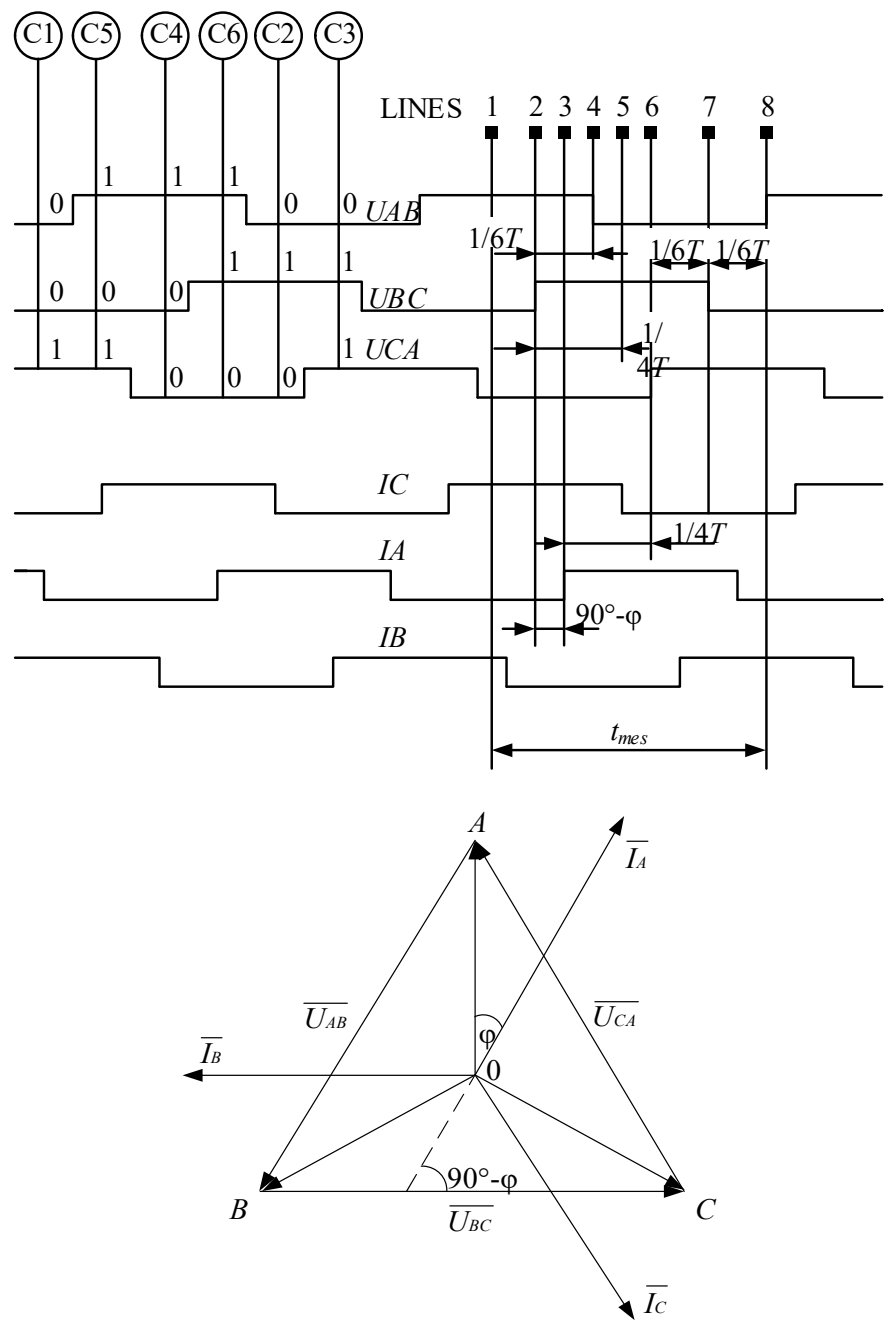

Fig. 1. Diagrams explaining the technique of multidimensional measurement of electricity parameters

To increase the speed of measurement of the active and reactive power of GSs, we shall refuse operations related to their calculations by the values and phase of the current. That could be achieved by forming a pulse on the load current measurement at the time of change in the state of bits of the specially selected voltage: for $I_{A} \rightarrow U_{B C}$; for $I_{B} \rightarrow U_{C A}$; for $I_{C} \rightarrow U_{A B}$. In this case (Fig. 1), the moments of measurement of active currents will be carried out along lines $2,4,6$, respectively, for currents $I_{\mathrm{A}}, I_{B}, I_{C}$. The practical possibility of applying this approach depends on the speed and principle of ADC operation.

As noted in [14], the further development of SAEPP is characterized by the expansion of the scope of using shaft-generators (SGs) by employing them not only in 
running modes but also in maneuver regimes. That leads to the formation of complex ship technological structures that combines the propulsion complex and the electric power system [15]. The desire to meet two criteria at the same time, reliability and cost-effectiveness, in such complexes is associated with frequent changes in their structure [16]. And the implementation of such operations as the start, stop, and the synchronization of diesel-generator (DG) to SG involves the participation of operators [17]. This circumstance causes the need to conduct research into the ergatic control systems taking into consideration the principles of ergonomics, which corresponds to the general methodology of formalization, construction, and solving complex systems and control tasks [18].

When solving the tasks of optimal control over SAEPP, the most difficult is to synthesize the laws of adaptive control over the process of SG synchronization with the grid under the mode of the irregular swing of the vessel [19]. Therefore, there is a need to solve problems in two statements - deterministic and stochastic [20].

The deterministic setting of synchronization process management tasks assumes the presence of a uniform change in the parameters of the movement of synchronized objects with their stable movement along specified trajectories [21]. That is when the latter are represented only by DG and the load on SAEPP almost does not change. The stochastic setting of synchronization tasks requires a study of the trajectory of the object of synchronization with stochastic fluctuations regarding its task caused by the influence of external factors (an irregular swing of the vessel or change of load) [22]. To detail the task of building an MP-system for managing the synchronization of generators, it is necessary to use the defined principles of system analysis. GS, which is directly controlled during synchronization, is called a synchronized object $S Y$. The synchronized object $O S$ is understood as a single or equivalent generator, the group of parameters of which during synchronization sets the trajectory of movement of synchronized objects [23].

On the one hand, the improvement of the synchronization process is carried out by developing and substantiating the algorithms of input information and control [24], solving management problems based on the principles of adjustment [25] with the analysis of the characteristics of the exchange processes between the object and computer [26]. On the other hand, due to the stochastic setting of the synchronization task [27] and checking the interaction of the entire complex of hardware and software tools [28].

For example, if the set of elements $S$, forming an automated technological process of synchronization, is represented as a combination of two subsets $S=S_{Y} \mathrm{U} S_{T P}$, then $S_{Y}$ are the elements of the control system, $S_{T P}$ are the elements of the technological process of synchronization. That is, these elements must be displayed in the form of the following ordered sets:

$$
\begin{aligned}
& S_{T P} \subset S_{O S}\left(N_{l}\right) \times S_{S Y}\left(N_{m}\right), \\
& S_{O S}\left(N_{l}\right)=\bigcup_{j=1}^{\left|N_{l}\right|} S_{O S}(j), \quad\left|N_{l}\right|=2^{l}-1, \\
& S_{S Y}\left(N_{m}\right)=\bigcup_{i=1}^{\left|N_{m}\right|} S_{S Y}(i),\left|N_{m}\right|=m,
\end{aligned}
$$

where $S_{O S}$ is the set of components (GS, SG) forming the object of synchronization, $S_{Y}\left(N_{m}\right), S_{S Y}\left(N_{m}\right)$ is the set of components that form synchronized objects; $S_{O S}(j)$ is the $j$-th GS composition, connected to buses and thus forming an object of synchronism; $S_{S Y}(i)$ is the GS with the $i$-th serial number, $i \in N_{m}$, prepared for synchronization; $j \in N_{i}, N_{i}$ is the numbering of GS composition operating on buses until the moment of synchronization; $l$ is the number of GSs connected to the main switchboard (MSB) bus.

We shall match the sets $S_{O S}\left(N_{l}\right)$ and $S_{S Y}\left(N_{m}\right)$ to the sets of predicates $S T S_{O S}\left(N_{l}\right)$ and $S T S_{S Y}\left(N_{m}\right)$, relating them through the following mutually unambiguous correspondence

$$
\left|S_{\text {OS }}\left(N_{l}\right)\right|=\left|\operatorname{STS}_{\text {OS }}\left(N_{l}\right)\right|
$$

and

$$
\left|S_{S Y}\left(N_{m}\right)\right|=\left|S T S_{S Y}\left(N_{m}\right)\right| .
$$

At the same time, $S_{O S}(j)=N S T S_{O S}(j)=I$, if the $j$-th generator composition is connected to the buses, and $S T S_{S Y}(i)=I$, if the $i$-th GS is ready for synchronization [29].

And then there is the task to determine a set of discrete and analog parameters and their corresponding converters necessary for the formation of the set of independent variables $X\left(N_{x} N_{m}\right)=\mathrm{U} X(j, i)[30]$.

Consequently, the SAEPP MP-system should include such $P R_{O S} P R_{S Y}$ converters that enable the following transitions

$$
S_{O S}\left(N_{l}\right) \stackrel{P R_{O S}}{\longrightarrow} S T S_{O S}\left(N_{l}\right)
$$

and

$$
S_{S Y}\left(N_{m}\right) \stackrel{P R_{S Y}}{\longrightarrow} S T S_{S Y}\left(N_{m}\right) .
$$

Information about the state of synchronized objects [31] should be classified according to three features: time scale frequency $-T_{T Z}$ of time character (analog/discrete) and the principle of fixation (interviewed/registered). Given the properties of the synchronization process, information about its progress received from sensors $d(1, i), \ldots, d(9, i)$, is classified as permanent, with a recovery frequency not more than $T_{T Z}$ (some recommendations for selecting the value for $T_{T Z}$ are given in [32]). Based on the second attribute, information about synchronized objects will be attributed to the analog average level (AC): generator load current $d(7, i),(d(8, i))$; generator voltage $d(4, i), d(5, i), d(6, i)$; generator current violation $d(3, i)$.

At the first stage of analysis, it is possible to use the language of resultant functions, widely used in the theory of algorithms [33]. That makes it possible to describe a number of transducers that generate predicates and dependent variables that underlie the functioning of a control system. The formation of predicates $I N C(i)$ та $D C R(i)$, determining the direction of adjustment of the synchronized object, taking into consideration the assessment of the synchronization process status at frequency $\left\{\omega_{\sin } \leq \omega_{s} \leq \omega_{s \max }, \omega_{s}>0\right\}$ is described as follows [34]:

$$
\begin{aligned}
& \bigcup_{I N C}\left(F R Q(i), F R S(i), P_{I N C}, t\right) \cong \\
& \cong(\overline{F R S}(i) \vee F R S(i) \& \overline{F R Q}(i)) \rightarrow I N C(i) ;
\end{aligned}
$$




$$
\begin{aligned}
& \cup_{D C R}\left(F R Q(i), F R S(i), P_{D C R}, t\right) \cong \\
& \cong(\overline{F R Q}(i) \& F R S(i)) \rightarrow D C R(i),
\end{aligned}
$$

where $I N C(i)$ and $D C R(i)$ are the predicates, and such that $I N C(i)=I$, if an increase in speed is required, and $D C R(i)=I$, if a decrease in speed is required for the $i$-th GS; $P_{I N C}$ and $P_{D C R}$ are converter algorithms, represented explicitly in the form of logical formulae.

The resultant function of the converter, which calculates the duration of the fitting pulse $Y_{I M E}(i)$, can be recorded as:

$$
\begin{aligned}
& Y_{I M P}\left(Y(2, i), Y_{C R}(i), P_{I M P}, t\right) \cong \\
& \cong Y_{C R}(i) \& Y(2, i) \stackrel{C_{N V}}{\longrightarrow} Y_{I M P}(i),
\end{aligned}
$$

where $Y(2, i)$ is the digital code of the sliding period, and $Y_{C R}(i)=f_{1}\left(s, \tau_{3}, n_{\text {out }}\right)-Y_{C R}(i)$ are the functional value characterizing the dynamic properties of the adjustment chain [35] at the time of synchronization, and $s$ is the rate of change in the rotational speed; $\tau_{3}$ is a delay in speed control channel; $n_{\text {out }}$ is the advance in the rotation frequency when the control signal is disabled.

However, in cases of complete imposition of time intervals, the marked pattern may be violated, which would lead to an error in determining the sign [36]. For a given case, the value of $\tau_{3}$ is considered minimal, therefore, to exclude possible errors, the measured values must be checked according to the condition $\tau_{3}>$ time constant of the synchronized object advance [37].

If one forms the Cartesian products of sets in the form of

$$
\begin{aligned}
& C H_{O S}=S T S_{O S}\left(N_{l}\right) \times S_{G S}\left(N_{m}\right) ; \\
& C H_{S Y}=S T S_{S Y}\left(N_{m}\right) \times S_{G S}\left(N_{m}\right),
\end{aligned}
$$

then the state of the synchronized objects, depending on the level of abstraction, can be described in more detail, using for this purpose information from the sensors of elements $G S\left(N_{m}\right)$ [38]. The level of abstraction will be determined by the management task solved. Formally, the problem is represented as determining the vector of structure parameters $C H S=\left(C H S_{O S}, C H S_{S Y}, C H S_{A P}, C H S_{M E}\right)$. This structure characterizes the composition of system devices, as well as connections - between them and the vector of control techniques $M C=\left(M C_{1}, \ldots, M C_{K}\right)$. The latter describes the problem of solving management tasks limited by the parameters of $P B M=\left(P B M_{1}, \ldots, P B M_{N}\right)$, tasks, which should provide the maximum value of the efficiency criterion $K=\left(K_{1}, \ldots, K_{M}\right)$, that is:

$\Pi P K \approx U_{K}(C H S, M C, P B M, P, t) \rightarrow \max K$.

\section{The aim and objectives of the study}

The aim of this study is to resolve the issue related to solving management tasks limited by the task parameters $P B M=\left(P B M_{1}, \ldots, P B M_{N}\right)$ as a totality of solutions to the set of interdependent tasks to ensure rapid control over synchronized objects. That would make it possible to change the parameters of generator assemblies under load in the conditions of external disturbances, and minimize the number of failed synchronizations.
To accomplish the aim, the following tasks have been set:

- to improve, based on the analysis of qualitative chara acteristics, the synchronization process of generator assemblies, with the development and substantiation of algorithms for processing input information and control;

- to resolve the task of optimal control over the frequency adjustment of the synchronized object and to choose an MPkit with determining the characteristics and actual capabilities of the selected MP-kit for the construction of SAEPP ACS or an assembled microprocessor complex (AMPC);

- to solve the task of high-speed control over the frer quency of synchronized objects, based on the principles of adjustment at the deterministic statement of the synchronization task, to analyze the characteristics of exchange processes between the object and computer, and to design pairing devices that are currently lacking, or that would be rational based on a series of criteria;

- to determine the moment of enabling generator mam chines at the stochastic setting of the synchronization task, debugging the software part, testing, and checking the interaction within the entire complex of hardware and software tools.

\section{Improving the synchronization process of generator assemblies}

Consider solving the task to synthesize an algorithm for managing the measurement process (Fig. 1). We believe that in MP-system information about parameters $\left(I_{\mathrm{A}}, I_{B}\right.$, $I_{C}, U, f$, and $\varphi$ ) is provided cyclically (at period $T_{T Z}$ ) and is related to the ADC control and operation. For this reason, the effectiveness of the proposed technique and the structure of measurement process control will depend on the speed and accuracy of the conversion of the ADC used, that is, the method and element base of conversion.

Linear voltages of the generator are selected as base values when determining the initial state. For an $n$-phase system, the number of possible system states is $\mathrm{C}_{P L o d}=2 n$ for the odd number of phases and is equal to $\mathrm{C}_{\text {PLev }}=n$ if the number of phases in the system is even. In turn, the number of impossible states in an $n$-phase system is determined by the expression $\mathrm{C}_{I L o d}=2^{n}-2 n$ for the odd number of phases, and $\mathrm{C}_{I L e v}=2^{n}-n-$ for even. Since the system is three-phase, the number of possible states of discrete signals reflecting the sinusoid half-periods is equal to six. Unambiguous sets $0-0-0$ and $1-1-1$ are not possible in a symmetrical three-phase system. Possible sets of states for alternating linear voltages $U_{A B}, U_{B C}, U_{C A}$ are denoted as $\mathrm{C}_{1}, \ldots, \mathrm{C}_{6}$ so that the ordinal state's number coincides with its binary code, that is, $\mathrm{C}_{1}-001\left(U_{A B}=0 ; U_{B C}=0 ; U_{C A}=1\right)$; $C_{2}-010 ; C_{3}-011 ; C_{4}-100 ; C_{5}-101 ; C_{6}-110$. Each state determines the only path (algorithm) that is possible according to the performance criterion for solving the measurement problem.

For example, if the source state is $\mathrm{C}_{4}$ (in the diagram, it is indicated by line 1), so the nearest time-dependent change is a change in the state of the $U_{B C}$ bit. Therefore, the measurement program determines when $U_{B C}$ jumps from " 0 " to "1" (line 2) and records the entire original word, in which all bits except $U_{B C}$ and $I_{A}$ are masked.

Analysis of the values of these bits makes it possible to determine the direction of the generator power. At the same time, if $U_{B C}$ switches: 


$$
\begin{aligned}
& \left.\begin{array}{l}
\text { from } 0 \text { to } 1 \text {, and } I_{A}=0, \\
\text { from } 1 \text { to } 0 \text {, and } I_{A}=1
\end{array}\right\} \text { - generator mode; } \\
& \left.\begin{array}{l}
\text { from } 0 \text { to } 1 \text {, and } I_{A}=1, \\
\text { from } 1 \text { to } 0 \text {, and } I_{A}=0
\end{array}\right\}- \text { motion mode. }
\end{aligned}
$$

Next, we shall count the single time intervals from line 2 to the moments of change in the status of the $I_{A}$ and $U_{B C}$ $\mathrm{C}$ bits (lines 3 and 4, respectively). The resulting numeric codes make it possible to determine the value of the phase angle $\varphi \varphi$ and the $T$ period. In this example, the digital interval code (line 2 - line 3 ) corresponds to the value $\left(90^{\circ}-\varphi\right)$, and the interval code (line 2 - line 4) corresponds to the value $1 / 6 T$. This information makes it possible to determine the moments of measurement of amplitude voltage values of $U_{B C}$ and phase currents $I_{\mathrm{A}}, I_{B}, I_{C} I_{\mathrm{A}}, I_{B}, I_{C}$ (respectively, on lines $5-8$ ), arising one by one at the interval $\tau=1 / 6 T$.

For the case shown in the diagram, the moment of measurement of the $I_{A}$ current amplitude is determined by the equality of the number of single time intervals of $1 / 4 T+\left(90^{\circ}-\varphi\right)$ from the moment $U_{B C}$ switches from 0 to 1 .

The digital codes obtained in this way that correspond to the current values of $I_{\mathrm{A}}, I_{B}, I_{C}, U, f$ and $\varphi$ make it possible to calculate the active $-P$, reactive $-Q$, and full power according to the known dependences: $P=U I \cos \varphi, Q=P \operatorname{tg} \varphi$, or $Q=U I \sin \varphi ; S=\sqrt{ } P^{2}+Q^{2}$, or $S=U I$, as well as determine the degree of unevenness of their distribution by phases (breakage, short circuit (SC), asymmetric load, etc.).

To describe the states of synchronized objects represented by the sets $C H S_{O S}$ and $C H S_{S Y}$, one needs to introduce the sets of independent $X\left(N_{x} N_{m}\right)$ and dependent $Y\left(N_{y} N_{m}\right)$ variables, where $N_{x}$ and $N_{y}$ number them. It is only natural that each variable $X(j, i)$ must correspond to a certain sensor $d(j, i)$, and, between them, there must be the converter $P=\{P R, C H V\}$ that ensures the following

$$
d(j, i) \stackrel{P(j, i)}{\longrightarrow} X(j, i) .
$$

We shall describe the set $X\left(N_{x} N_{m}\right)$ and the converters that form it, using the following scheme:

$$
\begin{aligned}
& d(1, i) \stackrel{C N V_{S W}}{\longrightarrow} X(1, i) ; \quad d(1, i) \in S W G(i) ; \\
& d(2, i) \stackrel{C N V_{\omega}}{\longrightarrow} X(2, i) ; d(2, i) \cong U(\omega), U(\omega)=k_{1} \omega_{D} ; \\
& d(3, i) \stackrel{C N V_{U}}{\longrightarrow} X(3, i) ; d(3, i) \cong U_{B}(G), U_{B}(G)=k_{2} I_{B} ; \\
& d(4, i) \stackrel{C N V_{U}}{\longrightarrow} X(4, i) ; d(4, i) \cong U(i)=U_{i} \sin \left(\omega_{i} t+\theta_{i}\right) ; \\
& d(5, i) \stackrel{C N V_{\omega}}{\longrightarrow} X(5, i) ; d(5, i) \cong U(i)=U_{i} \sin \left(\omega_{i} t+\theta_{i}\right) ; \\
& d(6, i) \stackrel{P R_{\theta}}{\longrightarrow} X(6, i) ; d(6, i) \cong U(i)=U_{i} \sin \left(\omega_{i} t+\theta_{i}\right) ; \\
& d(7, i) \stackrel{C N V_{I}}{\longrightarrow} X(7, i) ; d(7, i) \cong I(i)=I_{i} \sin \left(\omega_{i} t+\theta_{i}-\varphi_{i}\right) ; \\
& d(8, i) \stackrel{P R \varphi}{\longrightarrow} X(8, i) ; \quad d(8, i) \cong I(i)=I_{i} \sin \left(\omega_{i} t+\theta_{i}-\varphi_{i}\right) ; \\
& d(9, i) \stackrel{P R_{\delta}}{\longrightarrow} X(9, i) ; \quad d(9, i) \cong(i)=\left(\omega_{i} t+\omega_{c}+\delta_{0}\right),
\end{aligned}
$$

where $X(1, i), X(2, i)$ and $X(3, i)$ are the predicates, and such as $X(1, i)=I$, if the $i$-th GS is connected to the MSB bus; $X(2, i)=I$, if the $i$-th GS reached the rated rotations, that is, $\omega i=\omega_{N}, X(3, i)=I$, , if the $i$-th GS is excited, that is, $U_{B}(G)=U_{N} ; X(4, i), X(5, i), X(6, i), X(7, i), X(8, i), X(9, i)$ are the results of conversions by the $C N V$ hardware or $P R$ software techniques, represented as digital codes $\left(\omega_{c}-\right.$ synchronous frequency, $\delta_{0}-$ the initial angle of shift). These codes reflect, respectively, the amplitude values of linear voltages $X(4, i)$, frequency $X(5, i)$, shift angle between linear voltages $X(6, i)$, the amplitude values of phase currents $X(7, i)$, phase load angle $X(8, i)$ and the angle characterizing the rotor shift of the $i$-th GS in relation to the synchronous axis $X(9, i)$, without accounting for asymmetry to the moment of synchronization.

The resulting set of independent variables is not enough to fully describe the states of the synchronized synchronization object. We mean such characteristics as voltage difference, sliding frequency, the value of the difference in the angles of shear of rotors in relation to the synchronous axis, equal current, the duration of the synchronization process. We can obtain them by $C N V$ conversion on the sets $X\left(N_{x} N_{m}\right)$ and $X\left(N_{x} N_{l}\right)$ :

$$
\begin{aligned}
& \bigcup_{O S}\left(X(1,1), \ldots, X(1, m), P_{O S}, t\right) \rightarrow S T S_{O S}(j) ; \\
& \bigcup_{S Y}\left(X(1, i), X(2, i), X(3, i), P_{S Y}, t\right) \cong \\
& \cong(X(1, i) \& X(2, i) \& X(3, i)) \rightarrow S T S_{S Y}(i) ; \\
& \bigcup_{\Delta U}\left(X(4, i), X(4, j), P_{1}, t\right) \stackrel{P_{1}}{\longrightarrow} Y(1, i) ; \\
& Y(1, i) \cong \Delta U(i) ; \\
& \bigcup_{T S}\left(X(5, i), X(5, j), P_{2}, t\right) \stackrel{P_{2}}{\longrightarrow} Y(2, i) ; \\
& Y(2, i) \cong T_{S}(i) ; \\
& \bigcup_{\alpha}\left(X(9, i), X(9, j), P_{3}, t\right) \stackrel{P_{3}}{\longrightarrow} Y(3, i) ; \\
& Y(3, i), \cong \alpha(i) ; \\
& \bigcup_{\Delta I_{y}}\left(X(7, i), X(7, j), P_{4}, t\right) \stackrel{P_{4}}{\longrightarrow} Y(4, i) ; \\
& Y(4, i) \cong \Delta I_{y}(i) ; \\
& \bigcup_{T S Y}\left(C N T(S Y), P_{5}, \tau_{c}, t\right) \stackrel{P_{5}}{\longrightarrow} Y(5, i) ; \\
& Y(5, i) \cong T_{S Y} ; \\
& S T S_{O S}(j) \& S T S_{S Y}(i) \stackrel{P_{C N T}}{\longrightarrow} C N T(S Y),
\end{aligned}
$$

where $U_{O S}, U_{S Y}, U_{\Delta U}, U_{T S}, U_{\alpha}, U_{\Delta I y}, U_{T S Y}$ are the resultant functions: $P_{O S}, P_{S Y}, P_{C N T}, P_{1}, \ldots, P_{5}$ are the converter algorithms, and $P_{O S}, P_{S Y}$ and $P_{C N T}$ are represented explicitly, $P_{1}$ and $P_{2}$ are defined in $P$, and others, $P_{4}$ and $P_{5}$, are still in the implicit form; $S T S_{O S}(j) ; S T S_{S Y}(i)$ and $C N T(S Y)$ are the predicates, and such that if $S T S_{O S}(j)=I, S T S_{C S}(j)=N$, then the considered GS is treated as an object of synchronism, if $S T S_{S Y}(i)=I, S T S_{S Y}(i)=N$, then to the synchronized object, and if $C N T(S Y)=I$, then the process time counter is in operation; $Y(1, i), Y(5, i), Y(1, i), Y(2, i), Y(3, i), Y(4, i), Y(5, i)$ are the results of $P_{1}, \ldots, P_{5}$ transformations that generate, respectively: $Y(1, i)$ is the value of voltage difference $\Delta U(i)=U_{i}-U_{j}$; $Y(2, i)$ is the value of the sliding period $T_{S}(i)=1 / \omega_{S}(i) ; Y(3, i)$ 
is the value of the difference in the angles of shear of rotors of the synchronized objects in relation to the synchronous axis $\alpha_{i}=\delta_{i}-\delta_{j} ; Y(4, i)$ is the value of equal current when connecting the $i$-th GS to the object of synchronism $\Delta I_{y}(i)$ and $Y(5, i)$ is the current time of the synchronization process ( $T_{S Y}$ in digital codes).

Obviously, to assess the current state of the process of synchronization of GS, it is necessary to introduce a series of boundary (reference) values of the parameters of this process in the form of a set of constants $K\left(N_{K}\right)$

$$
\begin{aligned}
& K\left(N_{k}\right) K_{1} \cong \Delta U_{d o p} ; \quad K_{2} \cong 0 ; \quad K_{3} \cong T_{\text {smin }}, \\
& K_{4} \cong T_{\text {smax }} ; \quad K_{5} \cong \alpha_{d o p} ; \quad K_{7} \cong T_{\text {SYdop }},
\end{aligned}
$$

where $\Delta U_{d o p}, 0, T_{s \min }, T_{s \max }, \alpha_{d o p}, \Delta I_{y d o p}, T_{S Y d o p}$ are the valid and limit values of physical quantities in relative and absolute units, $K_{1}, \ldots, \ldots, K_{7}$ are their digital equivalents.

Converters $P$, assessing the state of the synchronization process, match the following resultant functions:

$$
\begin{aligned}
& \bigcup_{V L T}\left(Y(1, i), K_{1}, P R_{V L T}, t\right) \cong \\
& \cong Y(1, i) \exists K_{1}\left(P R_{V L T}\left(Y(1, i) K_{1}\right)\right) \rightarrow V L T(i) \text {; } \\
& \Delta U \leq \Delta U_{d o p} \\
& \bigcup_{S F R}\left(Y(2, i), K_{2}, P R_{S F R}, t\right) \cong \\
& \cong \bigcup_{S F R}\left(Y(2, i), K_{2}, P R_{S F R}, t\right) \cong \\
& \cong \forall Y(2, i) \exists K_{2}\left(P R_{S F R}\left(Y(2, i) K_{2}\right)\right) \rightarrow \operatorname{SFR}(i), \\
& \forall Y(2, i) \exists K_{2}\left(P R_{S F R}\left(Y(2, i) K_{2}\right)\right) \rightarrow S F R(i), \\
& T_{s}>0, \\
& \bigcup_{F R Q}\left(Y(2, i), K_{3}, K_{4}, P R_{F R Q}, t\right) \cong \\
& \cong \forall Y(2, i) \exists K_{3} \exists K_{4}\left(P R_{F R Q}\left(Y(2, i), K_{3}, K_{4}\right)\right) \rightarrow F R Q(i) \cong \\
& \cong \forall Y(2, i) \exists K_{3} \exists K_{4}\left(P R_{F R Q}\left(Y(2, i), K_{3}, K_{4}\right)\right) \rightarrow F R Q(i), \\
& T_{s \min } \leq T_{s} \leq T_{s \max }, \\
& \bigcup_{P H S}\left(Y(3, i), K_{5}, P R_{P H S}, t\right) \cong \\
& \cong \forall Y(3, i) \exists K_{5}\left(P R_{P H S}\left(Y(3, i), K_{5}\right)\right) \rightarrow P H S(i), \\
& \alpha \leq \alpha_{d o p}, \\
& \bigcup_{T S Y}\left(Y(5, i), K_{7}, P R_{T S Y}, t\right) \cong \\
& \cong \forall Y(5, i) \exists K_{7}\left(P R_{T S Y}\left(Y(5, i), K_{7}\right)\right) \rightarrow T S Y(i), \\
& T_{S Y} \leq T_{S Y d o p} .
\end{aligned}
$$

Thus, the use of the sets of $X$-independent and $Y$-dependent variables, as well as our synthesis of the mathematical models of converters $P$ for the information of the synchronization process, have made it possible to form the sets $C H S_{O S}=\left(X(1, i), \ldots . . X(9, i), Y(1, i), \ldots, Y(5, i), C H S_{S Y}=\right.$ $=\left(X(1, i), \ldots, X(9, i)\right.$, and a set of predicates $S T S_{T P}=(V L T(i)$, SFR(i), FRQ(i), PHS(i), TSY(i)), describing the state of the object of synchronism, the synchronized object, and the technological synchronization process, respectively.

The predicates $I N C(i), D C R(i)$ (1), obtained in this way, and the dependent variable $Y(I M E)$ make it possible to determine the functions that form the predicates that assign control influences RTI $(i)$ - "increase", RTD $(i)$ - "decrease" the frequency of the synchronized object, that is,

$$
\begin{aligned}
& \bigcup_{R T I}\left(I N C(i), F_{C N V}, Y(I M P), \tau_{0}, P_{R T I}\right) \rightarrow R T I(i) ; \\
& \bigcup_{R T D}\left(D C R(i), F_{C N V}, Y(I M P), \tau_{0}, P_{R T D}\right) \rightarrow R T D(i),
\end{aligned}
$$

where $F_{C N V}$ is the function for controlling the flow time of the adjustment pulse with the discreteness of $\tau_{0}$; its computational algorithm takes the following form:

$$
\bigcup_{C N V}\left(Y(I M P), \tau_{0}\right) \cong(\Delta Y(I M P) t-1(t+1)=0),
$$

moreover, $t$ is the previous, $t+1$ is the current run of technological cycles.

The resultant function $U_{P N T}$, which determines in the digital form the time of the synchronization optimum point expectation $-Y_{I M E}(i), Y_{P N T}$ by the parameters $X(5, j), X(5, i)$, $Y(5, i)$ is recorded in the implicit form for the time being:

$$
\bigcup_{P N T}=\left(X(5, j), X(5, i), Y(3, i), P R_{P N T}\right) \rightarrow Y_{P N T} .
$$

Knowing $Y_{P N T}$ and setting, while implicit, the value of the triggering time of the $Y_{S W}$ generator circuit, the predication function that determines the advance moment $A V D(i)$ is written as

$$
\bigcup_{A D V}=\left(\begin{array}{l}
Y_{S W}(i), Y_{P N T}, F_{T S T}, \\
Y_{P N T}, Y_{S W}(i), \tau_{0}, P_{A D V}
\end{array}\right) \rightarrow A D V(i),
$$

where $F_{T S T}$ is the function of timeout control with discreteness $\tau_{0}$. Its computational algorithm is:

$$
\begin{aligned}
& \bigcup_{T S T}\left(Y_{P N T}, Y_{S W}(i), \tau_{0}\right) \cong \\
& \cong(\Delta Y(P N T) t-1(t+1)=0) \rightarrow S W(i),
\end{aligned}
$$

where $Y_{S W}(i)=f_{2}\left(\tau_{A B}, \tau_{p 1}, \tau_{p 2}, \tau_{c s}\right)$, moreover, $\tau_{A B}, \tau_{p 1}, \tau_{p 2}, \tau_{c s}$ is the natural time of operation of the main elements of the generator switch enabling, respectively: control system $\left(\tau_{c s}\right)$ of the galvanic interchange relay $\left(\tau_{p 1}\right)$, the intermediate relay $\left(\tau_{p 2}\right)$, the generator circuit breaker $\left(\tau_{A B}\right)$.

Thus, assuming we obtained a complete set of the predicates $V L T(i), F R S(i), F R Q(i), P H S(i), A D V(i)$, then the resultant function generating the final predicate of the process $S W G(i)$ is written in an implicit form:

$$
\begin{aligned}
& \cup_{S W G}\left(\begin{array}{l}
V L T(i), Y F R(i), F Q R(i), \\
P H S(i), A V D(i), P_{S W G}, t
\end{array}\right) \cong \\
& \left(\begin{array}{l}
V L T(i) \& F R S(i) \& F R Q(i) \& \\
\& P H S(i) \& A V D(i)
\end{array}\right) \rightarrow S W G(i) .
\end{aligned}
$$

The analysis of the derived resultant functions shows that they can be grouped by the type of computational model and simplify their programming. With this approach, the 
vector of synchronization process management $M C=\left(M C_{1}\right.$, $\left.M C_{2}, M C_{3}, M C_{4}\right)$ is defined as follows:

$$
\begin{aligned}
& M C_{1}=\left(P R_{V L T}, P R_{F R S}, P R_{F R Q}, P R_{P H S}, P R_{T S V}\right) ; \\
& M C_{2}=\left(P_{I N C}, P_{D C R}, P R_{I M P}\right) ; \\
& M C_{3}=\left(P_{P N T}, P_{A D V}\right) ; \\
& M C_{4}=\left(P_{R T I}, P_{R T D}, P_{S W G}\right) .
\end{aligned}
$$

Implementation of the $P R_{V L T}, P R_{F R O}, P R_{F R S}, P R_{P H S}$ algorithms is related to the execution of arithmetic operations - deduction and comparison of the result with zero code. For example, a fragment that implements the $P R_{V L T}$ algorithm is:

$$
\begin{aligned}
& \text { lft } z_{1}=k_{1}-Y_{1}(i) \\
& \text { if } \quad z_{1}>0 \text {, then } 5 \\
& \text { let } V L T=0 \\
& \text { print "Voltage synchronization condition not met" } \\
& \text { let } V L T=1 .
\end{aligned}
$$

The $P_{I N C}, P_{D C R}, P_{A D V}, P_{R T D}$, and $P_{S W G}$ algorithms, obtained in the form of Boolean expressions, could be implemented both by software and hardware. The synthesis of the $P R_{I M E}, P R_{P N T}$ algorithms, associated with determining the functionalities $Y_{I M E}(i)=f_{1}\left(T_{s}, s, \tau_{3}, n_{\text {out }}\right)$ and $Y_{S W G}(i)=f_{2}\left(\tau_{A B}\right.$, $\left.\tau_{p 1}, \tau_{p 2}, \tau_{c s}\right)$, requires a separate study. For this purpose, we use the principle of relative movement of objects. The principle of relative movement of objects makes it possible to distinguish from the set $C H S$ the subsets of movements $C H S_{O S}^{T}$ and $C H S_{S Y}^{T}$ that characterize the trajectories of the object of synchronism and a synchronized object, respectively. Now, the main tasks of managing the synchronization process could be formulated in the following form: guide the synchronized object to the predefined trajectory of movement $C H S_{O S}^{T}$ and determine the moment of coincidence of the trajectories of the synchronized object CHSTSY with the assigned trajectory $\mathrm{CHS}_{O S}^{T}$.

To represent $C H S_{O S}^{T}$ and $C H S_{S Y}^{T}$ explicitly, we shall separate from them the groups of parameters $\{X(4, j), X(3, j)$, $X(9, j)\},\{X(4, j), X(5, j), X(9, j)\}$ and continue to consider their physical equivalents $\left\{U_{O S}, \omega_{O S}, \delta_{O S}\right\}$ та $\left\{U_{S Y}, \omega_{S Y}, \delta_{S Y}\right\}$ that fully characterize the trajectories of the movement of synchronization objects.

Solving these tasks is largely determined by the patterns laid down in the task setting itself: deterministic and stochastic.

The deterministic setting of synchronization process management tasks assumes the presence of a uniform change in the movement parameters of synchronized objects at their stable movement along the specified trajectories, that is,

$$
\frac{d \omega_{S Y}}{d t}=\text { const } ; \frac{d \omega_{O S}}{d t}=\text { const } ; \frac{d \omega_{S}}{d t}=\text { const }
$$

or, at the digital level

$$
\frac{\Delta X(4, i)}{\tau}=\text { const; } \frac{\Delta X(4, j)}{\tau}=\text { const; }
$$

$$
\frac{\Delta Y(5, i)}{\tau}=\text { const }
$$

At the same time, the assumed task to guide a synchronized object to the assigned motion trajectory $\omega_{3}=f\left(\omega_{O S}\right)$ can be considered using a mathematical model. This model includes differential equations for describing the operation of the GS generator, the regulator $R G$, and the $G M$ regulator servo motor, as elements of adjusting the control task according to the parameter $\omega_{S Y}$ :

$$
\begin{aligned}
& \left(T_{G S} p+1\right) \omega^{*}=k_{G S} l^{*} ; \\
& \left(T_{1} p^{2}+T_{2} p+1\right) l^{*}=k_{R G}\left(T_{3} p+1\right) \omega^{*} ; \\
& p \omega_{3}=k_{G M} t_{I M P},
\end{aligned}
$$

where $\omega^{*}=\frac{\omega_{S Y}-\omega_{3}}{\omega}$ is the relative change in the current value of the angular rotation frequency $-\omega_{S Y}$ compared to the previously set value $\omega_{3} ; l^{*}=\frac{l-l_{0}}{l_{H}-l_{X}}$ is the relative change in the position of the coupling of the regulator, while $l_{0}$ is the position in the previous state, $l_{X}$ - under an idle mode, $l_{H}$ - at a rated load; $k_{G S}, k_{R G}, k_{G M}$ are the gain coefficients of $\mathrm{GS}$, regulator, and servo motor; $T_{G S}, T_{1}, T_{2}, T_{3}$ are the time constants of GS and regulator.

However, the above system of equations (5) is not suitable for solving management tasks related to determining the moment of coincidence of trajectories of the synchronized objects: the characteristics of the movement of synchronized objects should be considered in mutual dependence because a change in the space of the eponymous phases of electromagnetic fields of the synchronized objects, represented in the form of vectors, at any given time $t$ is determined in the polar coordinate system. Therefore, the vector equation of the movement of a synchronized object regarding a synchronization object can be recorded in the following form:

$$
\bar{r}_{S Y}^{(k)}=\bar{r}_{O S}^{(k)}+\bar{r}_{S}^{(k)},
$$

where

$$
\bar{r}_{S Y}^{(k)} \cong\left|u_{S Y}^{(k)}\right|=\bigcup_{S Y} \sin \omega_{S Y} t^{(k)}-,
$$

is the radius vector that reflects the position of a synchronized object in space at the $k$-th moment in time;

$$
\bar{r}_{O S}^{(k)} \cong\left|u_{O S}^{(k)}\right|=\bigcup_{O S} \sin \omega_{O S} t^{(k)}-,
$$

is the radius vector that reflects the position of the object of synchronism in space at the $k$-th moment in time;

$$
\bar{r}_{S}^{(k)} \cong\left|u_{S}^{(k)}\right|=\bigcup_{O S} \bigcup_{S Y} \sin \frac{\omega_{S O}-\omega_{S Y}}{2} t^{(k)}-,
$$

is the vector that determines the value that bends the voltage at the $k$-th moment in time.

The hypothesis of uniform change in the parameters of the movement of synchronized objects under real conditions is expedient only for a certain time marked as $d_{i s t}$. Thus, this demand must satisfy changes in the movement parameters of synchronized objects in the interval between the recovery of information data and describe a vector of structure $\mathrm{CHS}$ 
parameters. That is, these expressions are valid in the case $\tau<d_{\text {ist }}$, where $\tau \approx T_{T Z}=t^{(k)}-t^{(k-1)}$ is the period of data recovery in MP-system. Obviously, the $T_{T Z}$ period should be chosen on the basis of the conditions of both technical and operational capabilities (speed characteristics, electromagnetic and electromechanical time constants, the inertial properties of drive engines, etc.), and the ability of MP-system to process the existing flow of information taking into consideration the adaptation of the human operator to its perception and the speed of producing solutions. The service time (processing) $t$ depends on the functions of the service time allocation functions $F(t)$ for the existing technical means of automation of the synchronized object and MP-system in general. Knowing $F(t)$, one can determine the probability of not exceeding the service time $t$ of some given value $t_{r e f}=T_{T Z}-t_{P R}$ :

$$
P\left(t_{r e f}\right)=f(F(t)) ; t \leq t_{r e f},
$$

and the average service time of one cycle $M(t)=\int_{0}^{\infty} t_{P R} \mathrm{~d}(F(t))$, where $t_{P R}$ is the time allotted to work with the current program.

Thus, the organization of control over synchronized objects in MP systems should provide for cyclical recovery at period $T_{T Z}$ and the processing of information data (directly during the control process, depending on its technological characteristics and properties of the control system). In addition, the deterministic setting of synchronization tasks leads to the need for more careful control over the movement parameters of synchronized objects. In case of deviation of their movement from the specified trajectories, in accordance with the adopted hypothesis, it requires a secondary solution to the problem with the subsequent adjustment of movement parameters.

The tasks of synchronization in a deterministic setting are the least complex and most convenient in cases where there are low-frequency changes.

The proposed stochastic approach to solving synchronization problems makes it possible to investigate the process of synchronization when the object of synchronism moves along the predefined trajectory with stochastic fluctuations regarding its setting under the influence of external factors. There is an obvious difficulty in implementing the task of managing a synchronized object when it moves on an unforeseen trajectory with random parameters, for example, in case of failure of the drive engine or its controls.

The system of equations describing the movement of the synchronized object is complemented by a set of stochastic characteristics of the control task, that is,

$$
\begin{aligned}
& \left(T_{G S} p+1\right) \omega^{*}=k_{G S} l^{*} ; \\
& \left(T_{1} p^{2}+T_{2} p+1\right) l^{*}=k_{R G}\left(T_{3} p+1\right) \omega^{*} ; \\
& p \omega_{r e f}=k_{G M} t_{I M P} ; \\
& \bar{r}_{S Y}^{(k)}=\bar{r}_{O S}^{(k)}+\bar{r}_{S}^{(k)} ; \\
& X \omega_{O S}(t)=\left(M \omega_{O S}(t), D \omega_{O S}(t), K \omega_{O S}(\tau)\right),
\end{aligned}
$$

where $M \omega_{O S}(t), D \omega_{O S}(t)$ and $K \omega_{O S}(\tau)$ is the mathematical expectation, variance, and correlation function of random value $X \omega_{O S}$, describing external perturbation by the rotation frequency of the object of synchronism.

The system of scholastic equations obtained in this way makes it possible to solve synchronization problems taking into consideration the nature of external disturbances. In this case, the course of solving synchronization tasks, in general, can be described by the following algorithm scheme (FAS):

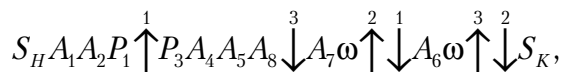

where $S_{H}$ and $S_{K}$ are the start and end operators; $A_{2}$ - determining the task of control and its scholastic characteristics; $A_{3}$ - determining the depth of the forecast; $A_{4}$ - forecasting changes in the task parameters; $A_{5}$ - adjustment of the control task; $A_{6}$ and $A_{8}-$ solutions to the management task in the deterministic and scholastic statements; $A_{7}$ - derivation of control commands; $P_{1}$ - checking the condition of the need for a stochastic approach to solving problems; $A_{1}$ - data entry.

Thus, the tasks to manage the synchronization process, taking into consideration the patterns in their statements, make it possible to describe, in a general form, the vector $\mathrm{PBM}=\left(\mathrm{PBM}_{1}, \mathrm{PBM}_{2}, \mathrm{PBM}_{3}, \mathrm{PBM}_{4}\right)$. In this case, $\mathrm{PBM}_{1}$ and $\mathrm{PBM}_{3}$ - determining those control actions on GM that ensure guiding the synchronized object on the specified trajectory of movement in the deterministic and stochastic statements, respectively. $\mathrm{PBM}_{2}$ i $\mathrm{PBM}_{4}$ - determining the moment of enabling SW in the deterministic and stochastic statements, respectively. A specific description of the PBM vector is possible at the established criteria for optimizing the tasks that determine its parameters.

The task of optimization will be connected to defining the law of control, which would satisfy the condition for an extremum as a quality indicator and would take into consideration the requirements of limiting control influences, the quality of electricity, as well as possible changes in the technical characteristics of synchronization objects. The latter requirement causes the need to consider the developed system in the class of adaptive control systems. Since the synchronization process is a complex and multi-critical task, it is advisable to decompose solving it - to consider independently, as a solution to the separate part of the task. To evaluate a particular task, one can select one criterion if the task is simple and small in scope, and a group of criteria if the task is complex. At the same time, one of the groups of criteria is chosen as the main one, while the others are additional and, in the conditions of their mutual antagonism, may have a priority basis. When solving private problems, a change in the main and additional criteria can be made, or a change in the priority and degree of the importance of additional criteria. That happens depending on the requirements for performance, real situation, type of proposed control, and other factors affecting the course of the synchronization process. Certain synchronization tasks are conditionally divided into performance tasks, that is, the task of control execution in the shortest possible time, the task of control in the predefined time, and the task of control under the proposed conditions. Thus, based on the meaning of the content of control tasks, we shall write three types of optimality criteria:

- for performance tasks

$$
K=\min _{u} T \text {, }
$$


where $T$ is the time of control; $u$ is the parameter of control over a synchronized object;

- for control tasks at a specified time

$$
K=\min \left(T_{r e f}-T_{\text {fact }}\right),
$$

where $\mathrm{T}_{\text {ref }}$ is the specified (proposed) time of the necessary control, $\mathrm{T}_{\text {fact }}$ is the actual time spent on the control task execution;

- for control tasks under specified conditions

$$
K=\min \left|\Delta P_{\text {dop }}-\Delta P_{\text {fact }}\right|,
$$

where $\Delta P$ is a valid deviation of the parameter $P_{i}$ from $P_{\text {dop }}$; $\Delta P_{\text {fact }}$ is the actual deviation of $P_{i}$ from $P_{d o p}$.

In accordance with the considered statements of tasks for managing synchronized objects (deterministic and stochastic), we shall introduce an optimality criterion based on (11).

The least difficult is a control task in the deterministic setting, which is based on the fact that the control over synchronized objects (and, therefore, the trajectories of their movement in space) is set by known functions of time. A separate case of such a statement allows that the voltage curves of the network UOS, as an object of synchronism, and the generator $U S Y$, as a synchronized object, are the sinusoidal functions of time (7), (8), respectively. Then the optimality criteria for synchronization tasks in the deterministic setting are fully described by dependences (13) to (15).

Since the $\left(P_{3.1}-P_{3.3}\right)$ conditions are decisive in solving the tasks of exact synchronization, then it is advisable to use expressions (1) to evaluate the effectiveness of control over this process. Determining $\Delta P_{d o p}$ and $\Delta P_{\text {fact }}$ is based on the trio of parameters $D=\left(\Delta U_{d o p}, \omega_{s d o p}, \alpha_{d o p}\right)$ and $\Phi=\left(\Delta U, \omega_{s}, \alpha\right)$, respectively. In general, taking into consideration that exceeding each of the parameters of the trio $\Phi$ of the corresponding tolerance with $D$ leads to a significant equalization current during synchronization, we shall match the difference $\left(\Delta P_{d o p}-\Delta P_{\text {fact }}\right)$ to the following functionality

$$
i_{u r}=\frac{\sqrt{2} K E_{d}^{\prime \prime} E_{d}^{\prime}}{x_{d 1}^{\prime \prime}+x_{d 2}^{\prime \prime}+x_{c}} \sin \frac{\alpha_{0}}{2},
$$

where $K$ is the coefficient that takes into consideration the aperiodic component of the current; $x_{d 1}^{\prime \prime}$ and $x_{d 2}^{\prime \prime}$ are the inductive scattering resistances along the longitudinal axis of synchronized generators; $x_{c}$ is the equivalent inductive resistance of network elements connecting synchronized generators; $\alpha_{0}$ is the initial phase shift angle between the longitudinal $E M F$ of synchronized generators, that is, between $E_{d 1}^{\prime \prime}$ and $E_{d 2}^{\prime \prime}$.

Thus, the main criterion for evaluating the effectiveness of synchronization process control takes the following form:

$$
K=\min _{u} i_{u r}
$$

The synchronization process leads to the need to analyze the characteristics of external perturbations. In a general case, two types of tasks for evaluating the effectiveness of actions are possible. The first is characterized by the probability that a certain effect will be achieved as a result of the performed actions, the second is based on determining the average value (mathematical expectation) of the expected effect. Applying the resulting system of stochastic equations to each of the two types of performance assessment tasks, we obtain the following two groups of optimality criteria.

For the tasks of type I:

$$
P R_{I M P}: Y_{I M P}(i) \rightarrow t_{I M P}-\text { for performance; }
$$

$K=\min _{u}\left(P\left(T_{\text {ref }}-T_{\text {fact }}\right)\right)-$ for execution within the specified time;

$$
K=\min _{u}\left(P\left(P_{r e f}-P_{\text {fact }}\right)\right)-\text { for execution at the predefined }
$$

For the tasks of type II:

$$
\begin{aligned}
& K=\min _{u}(M(T))-\text { for performance; } \\
& K=\min _{u}\left(M\left(T_{\text {ref }}-T_{\text {fact }}\right)\right)-\text { for execution within the spec- }
\end{aligned}
$$
ified time;

$K=\min \left(M\left(\Delta P_{\text {dop }}-\Delta P_{\text {fact }}\right)\right)-$ for execution within the specified time.

The first group of criteria will be used to assess the quality of system functioning under a stochastic approach to determining the time of a synchronization optimum.

The second group of criteria will be used to evaluate a system when managing the process of equalizing the frequencies of synchronized objects and determining the moment of supply of the proactive pulse to turn on the generator.

According to the requirement, according to which MP-system in the formation of control should take into consideration a change in the technical characteristics of objects, then these tasks should be solved on the basis of the classical theory of dual control by Feldbaum. Using the ideas of this theory, the synthesis of the control algorithm, for the proportion of cases under which the last two tasks fall, is reduced to the following.

Supposing that the known mathematical model (or there are experimental data) describes at discreteness $\tau$ a change in the value of $X(i, j) \in \mathrm{X}\left(N X_{1}-N_{m}\right)$ in the process of translation $-\mathrm{P}_{X}$ of the synchronized object, from one - the initial, to the other - the specified, final state, that is,

$$
\bigcup_{X}\left(X_{H}, \theta, \mathrm{Z}, P_{X}, \tau\right) \rightarrow X_{3}
$$

where $U_{X}$ is the resultant function, $X_{N}(\tau)$ is its value at the time the control influence appears; $\theta(\tau)$ i $Z(\tau)$ are the changes in the technical and operational performance of the synchronized object during operation. At the same time, in the process of implementing each of the translations of type $X_{N} \stackrel{P_{X}}{\longrightarrow} X_{r e f}$ at any $k$-th moment of time MP-system the proposed $-X_{k}(\tau)$ and the actual $-X_{k}^{\Phi}(\tau)$ value of the managed value are known. Information about parameters $Z(\tau)$ is set by the vector of observations $V_{k-1}=\left(V_{k-1}, V_{k-2}, \ldots, V_{N}\right)$ of the value $X_{N}(\tau)$. At any given time, the values $X_{N}(\tau)$, specified by the control vector $\theta_{k-1}=\left(\theta_{k-1}, \theta_{k-2}, \ldots, \theta_{N}\right)$, are recorded in the memory of an MP system and represent observable prehistory of the control process. Here, $V_{i}=X_{i}+h_{i}, i \in 0, k-1$, where $h_{i}$ is a random error in the measurement of the value $X_{i}$ with a known probability distribution density $P\left(h_{i}\right)$. 
If the observations of the implemented translation show that there is a deviation of the actual value $X_{k}^{\Phi}(\tau)$ of the managed value from the proposed $X_{k}^{\Pi}(\tau)$, , then the losses introduced by the system could be estimated by the specific loss function $W_{k}(\tau)=W\left(X_{k}^{\Phi}, X_{k}^{\Pi}, \tau\right)$. It is quite obvious that during the final time of the MP system functioning during the translation process, the generalized loss function will take the following form: $W=\sum_{k=0}^{n} W\left(X_{k}^{\Phi}, X_{k}^{\Pi}, \tau\right)$.

Thus, the system will be considered optimal for which the complete risk $R$ - the mathematical expectation of the loss function - is minimal, that is,

$$
M\{W\}=\sum_{k=0}^{n} M\left\{W_{k}\right\}=\sum_{k=0}^{n} R_{k} \rightarrow \min R .
$$

Here, $R_{k}$ is the specific risk that is defined as

$$
R_{k}=\int_{\psi\left(V_{k-1}, \theta_{k-1}\right)} r_{k} P\left(V_{k-1}, \theta_{k-1}\right) \mathrm{d} \psi
$$

The functionality $r_{k}$ in (17) is the conditional specific risk, it is a mathematical expectation of specific losses $W_{k}$ at the fixed values of vectors $V_{k-1}$ and $\theta_{k-1}$. It is determined as:

$$
Z_{k}=\int_{\psi\left(Z, \theta_{k}\right)} W\left(X_{k}^{\hat{O}}\left(\theta_{k}, Z\right) X_{k}^{P}\right) P\left(Z / V_{k-1}, \theta_{k-1}\right) S_{k} \mathrm{~d} \psi
$$

where $S_{k}=P\left(\theta_{k} / V_{k-1}, \theta_{k-1}\right)$ is the conditional density of the distribution $\theta_{k}$, called the specific control strategy. In (18) and (19), the character $\psi$ denotes the integration region. The expression $P\left(Z / V_{k-1}, \theta_{k-1}\right)$ is a posteriori density of the distribution of an unknown parameter $Z$. At given a priori densities $P_{0}(Z)$ and $P\left(h_{i}\right)$ are determined from the Bayesian formula:

$$
P\left(Z / V_{k-1}, \theta_{k-1}\right)=\frac{P_{0}\left(Z \prod_{i=0}^{k-1} P\left(V_{i} / Z, \theta_{i}\right) \prod_{i=0}^{k-1} S_{i}\right)}{P\left(V_{k-1}, \theta_{k-1}\right)} .
$$

The conditional density of the distribution $P\left(V, Z, \theta_{1}\right)$ is determined taking into consideration (16) based on the known density of the distribution $P\left(h_{i}\right)$. The sequence of functions at $\delta=\left\{S_{0}, S_{1}, \ldots, S_{n}\right\}$ is a control strategy. The dependence of risk $R$ on strategy $\delta$ is denoted $R_{\delta}$; it will be considered optimal if it minimizes the risk $R$. This strategy is searched in the class of valid strategies $\Delta$. From (18), (19), and (20), it follows that each term $R_{k}$ in (17) depends on the choice of the sequence $\left\{S_{0}, S_{1}, \ldots S_{n}\right\}$.

At the same time, the choice of a specific $S_{k}$ strategy affects not only $R_{k}$ at the $k$-th moment but also the value of all future specific risks $R_{k+1}, \ldots$, $R_{n}$. This effect is manifested through a posteriori density of the distribution of an unknown parameter and constitutes the essence of the duality of control: the choice of control determines not only the behavior of value $X$ but also the rate of accumulation of information about a change in $Z$.
Given the above, they conclude that the optimal strategy for managing the synchronization process is directly related to the development of effective ways to measure the technical indicators of control objects and determine their changes during operation, in particular during synchronization.

\section{Solving the task of optimal control over the frequency adjustment of the synchronized object}

The resultant function of the converter, which calculates the duration of the adjustment pulse $Y \operatorname{IME}(i)$ (3), was derived implicitly. Now, a solution to the set task will be related to determining the law of control

$$
P R_{I M P}: Y_{I M P}(i) \rightarrow t_{I M P}
$$

that makes it possible to calculate and form such influences $t_{I M P}$, the implementation of which in the adjustment chain $G M \rightarrow R G \rightarrow G S$ will ensure the transfer of the synchronized object to the specified trajectory of movement $\omega_{\text {ref }}$ over the minimum control time $t_{p}$.

In this regard, we shall highlight, in the structure shown in Fig. 1, the frequency adjustment control channel. We shall present it with the equivalent scheme of digital ACS with the output signal $\omega_{S Y}$ (Fig. 2). Here, an MP system acts as a discrete automatic control device that works cyclically, with the interval of information recovery $\tau=T_{T Z}$.

The deterministic statement (4) and (5) makes it possible to consider a control device as an element of continuous action, the law of functioning of which could be described mathematically. In a general case, this will be the dependence in the following form

$$
t_{I M P}=\frac{\omega^{*}}{W_{C R}(p)}
$$

where $W_{C R}(p)$ is the transfer function (TF) of the adjustment circuit; $p$ is a Laplace operator.

For the structure shown in Fig. 2, one can record:

$$
\begin{aligned}
& W_{C R}(p)=W_{G M}(p) \cdot W_{R G}(p) \cdot W_{G S}(p)= \\
& =\frac{k_{G M}}{p} \cdot \frac{k_{R G}\left(T_{3} p+1\right)}{T_{1} p^{2}+T_{2} p+1} \cdot \frac{k_{G S}}{T_{G S} p+1} \\
& W_{C R}(p)=\frac{k_{G M} k_{R G} k_{G S}\left(T_{3} p+1\right)}{T_{1} T_{G S} p^{4}+\left(T_{2} T_{G S}+T_{1}\right) p^{3}+\left(T_{2}+T_{G S}\right) p^{2}+p} .
\end{aligned}
$$

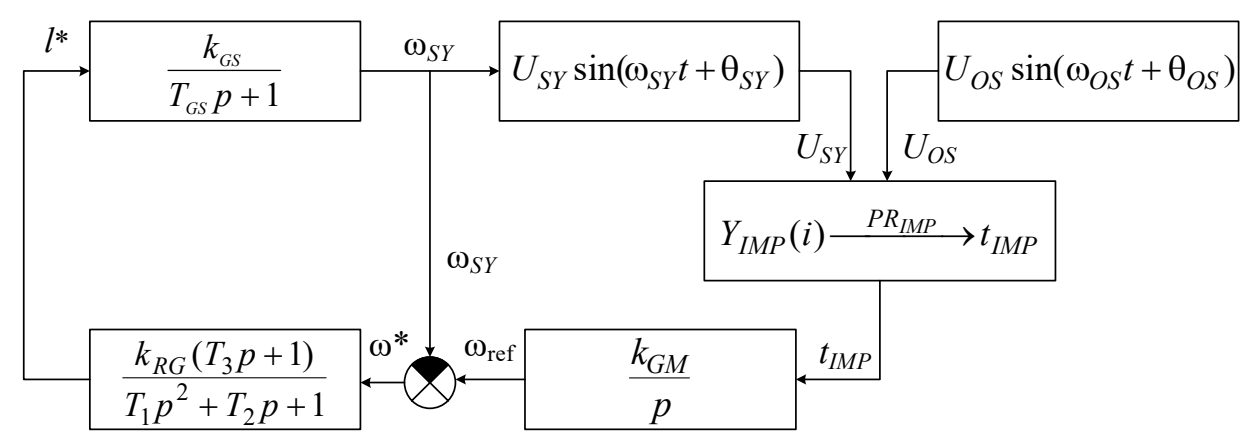

Fig. 2. Structural diagram of the frequency adjustment channel 
Thus, the transfer function

$$
\omega^{*}=\left[\frac{k_{G M} \cdot k_{R G} \cdot k_{G S}\left(T_{3} p+1\right)}{T_{1} T_{G S} p^{4}+\left(T_{2} T_{G S}+T_{1}\right) p^{3}+\left(T_{2}+T_{G S}\right) p^{2}+p}\right] t_{I M P},
$$

will describe the real transition process caused by the implementation in the channel of adjustment of the control influence $t_{I M P}$, while the inverse dependence

$$
t_{I M P}=\frac{\omega^{*}}{\left[\frac{k_{G M} \cdot k_{R G} \cdot k_{G S}\left(T_{3} p+1\right)}{T_{1} T_{G S} p^{4}+\left(T_{2} T_{G S}+T_{1}\right) p^{3}+\left(T_{2}+T_{G S}\right) p^{2}+p}\right]},
$$

may form the basis of the desired control.

For this reason, $W_{C R}(p)(23)$ is described by the trio of characteristics $B=\left(s, \tau_{\text {ref }}, n_{\text {out }}\right)$, which makes it possible to describe the desired control law by a dependence in the following form

$$
t_{I M P}=F_{2}\left(\omega_{\mathrm{s}}, \tau_{r e f}, s, n_{\text {out }}\right),
$$

where $s$ is the rate at which the $G S$ speed changes, $\tau_{\text {ref }}$ is the delay in a rotation frequency control channel, $n_{\text {out }}$ is the advance rotation frequency when the $t_{I M P}$ influence is removed.

The presented approach to determining $t_{I M P}$ makes it possible to take into consideration all possible changes in the parameters and dynamic properties that characterize the chain $G M \rightarrow R G \rightarrow G S: k_{G M}, k_{R G}, k_{G S}, T_{G S}, T_{1}, T_{2}, T_{3}$ during SAEPP operation. However, the implementation of a dependence of the following form in MP systems:

$$
t_{I M P}=F_{1}\left(\omega^{*}, T_{G S}, T_{1}, T_{2}, T_{3}, k_{R G}, k_{G M}, k_{G S}\right)
$$

is complex, and, most often, impossible without reliable determining the $T_{G S}, T_{1}, T_{2}, T_{3}, k_{G M}, k_{R G}, k_{G A}$ parameters in real SAEPP with a possible change in these parameters directly during synchronization.

That is, it is possible to write down

$$
t_{I M P}=\omega_{s}(\mathrm{H}) \cdot \operatorname{ctg} \gamma_{n}=\frac{\omega_{s}(\mathrm{H})}{s(M)}
$$

and, on the basis of

$$
\tau_{r e f}(M)=t_{p}-t_{I M P}=\frac{\omega_{s}(H)}{s(M)}\left(K_{n}-1\right)
$$

to define a calculation formula for determining the adjustment coefficient:

$$
K_{n}=\frac{\tau_{r e f}(M) \cdot s(M)}{\omega_{s}(H)}+1 .
$$

Fitting (27) in (28) produces:

$$
t_{p}=\frac{\omega_{s}(H)}{s(M)}+\tau_{z}(M)
$$

reflecting the dependence $t_{p}$ on the initial slip frequency $\omega_{s}(H)$ and the dynamic properties of the elements of the adjustment chain, represented by the set $s(M), \tau_{r e f}(M)$, $n_{\text {out }}(M)$. Taking into consideration the possibility of changing these values under the operational mode of SAEPP, or a change in $\omega_{S}$ due to the fluctuations in $\omega_{O S}$ directly during synchronization, we shall introduce into the law (algorithm) of adjustment control a conformity check, that is, compliance:

$$
\omega_{S Y}\left(t_{H}+\frac{1}{2} t_{p}\right) \cong \omega_{M}=\omega_{S Y}(H)+\frac{1}{2} \omega_{s}(H) .
$$

If, in this case, $\omega_{S Y=} \omega_{M}$, the transition

$$
\omega_{S Y}(H) \stackrel{t_{M P}}{\longrightarrow} \omega_{S Y}\left(K_{M}\right)
$$

is performed according to the specified trajectory. In cases where $\omega_{S Y} \neq \omega_{M}$, for example, $\omega_{S Y=} \omega_{N}$ or $\omega_{S Y}=\omega_{L}$, an appropriate correction of the influence $t_{I M P}$ is required, related to the calculation of $t_{I M P}^{M}$ according to the formula $t_{I M P}^{M}=t_{I M P} \pm \Delta t_{M}$, where the value $\Delta t_{M}=\left|\omega_{S Y}\left(t_{n}+\frac{1}{2} t_{p}\right)-\omega_{M}\right| \frac{K_{0}}{s(M)}$ sign is set as a result of checking any of the conditions $\omega_{S Y}>\omega_{M}$ or $\omega_{S Y}<\omega_{N}$.

Significant importance is the check of the condition $\omega_{O S}\left(t_{h}\right)=\omega_{O S}\left(t_{m}\right)$, which makes it possible to determine the course of the further solution to the task set. Thus, if $\omega_{O S}(t) \neq \omega_{O S}\left(t_{m}\right)$ and a change in $\omega_{O S}(t)$ is deterministic, then re-calculations of $t_{I M P}$ under the new initial conditions are required. In the case of stochastics changes in $\omega_{O S}(t)$, the law of adjustment control should be changed, for example, to form a constant signal $t_{I M P}$ until the following conditions are met:

$$
\begin{aligned}
& \omega_{S Y}(t)=\omega_{\text {ref }}(t)+n_{\text {out }}(M), \text { if } \omega_{S Y}(H)>\omega_{\text {ref }}(H) ; \\
& \omega_{S Y}(t)=\omega_{\text {ref }}(t)-n_{\text {out }}(M), \text { if } \omega_{S Y}(H)<\omega_{\text {ref }}(H),
\end{aligned}
$$

where $\omega_{\text {ref }}(t)=\omega_{O S}(t)+\left(\omega_{\text {smax }}-\omega_{\text {smin }}\right) / 2$ characterizes the specified adjustment trajectory.

The fulfillment of the condition $\omega_{O S}\left(t_{h}\right)=\omega_{O S}\left(t_{M}\right)$ indicates that the existing discrepancy between the actual $\omega_{S Y}\left(t_{H}+\frac{1}{2} t_{p}\right)$ and proposed $\omega(M)$ values is caused by a change in the dynamic properties of the adjustment chain, which requires determining and correcting the $s(M)$, $\tau_{\text {ref }}(M), n_{\text {out }}(M)$ values.

The organization of the adaptive automatic control system, which implements the proposed law of frequency adjustment, will be associated with the automation of the process of determining the $s, \tau_{\text {ref }}, n_{\text {out }}$ values and re-adjustment of $s(M), \tau_{r e f}(M), n_{\text {out }}(M)$ directly during the SAEPP operation.

The results of the registration obtained in this way make it possible to determine:

$$
s(M)=\frac{1}{n} \sum_{i=1}^{n} s(i) \text { and } s(i)=\frac{\Delta f(i)}{t_{I M P}(i)},
$$

when forming $t_{I M P} \in t_{I M P}^{S T}$ for increasing $s(I N C)$ and reducing $s(D C R)$ the frequency of rotations, as well as

$$
t_{p}(i)=t_{k}(i)-t_{h}(i)
$$

where $\Delta f(i)=\left|f_{h}(i)-f_{k}(i)\right|$ is the difference in the frequency of transition caused by the implementation of $t_{I M P}$, and $n$ is the number of implementations. 
With this approach, the $\tau_{r e f}(M)$ and $n_{\text {out }}(M)$ values can be calculated as follows:

$$
\begin{aligned}
& \tau_{\text {ref }}(M)=\frac{1}{n} \sum_{i=1}^{n} \tau(i), \\
& \tau_{\text {ref }}(i)=t_{p}(i)-t_{\text {IMP }}(i), \\
& n_{\text {out }}(M)=\frac{1}{n} \sum_{i=1}^{n} n_{\text {out }}(i), \\
& n_{\text {out }}(i)=\Delta f(i)-\left|f_{\mathrm{H}}(i)-f(i)\right|,
\end{aligned}
$$

where $f_{p}(i)$ is the value registered at the time of removal of the control influence $t_{I M P}$.

Given the above, the GS control algorithm at the automated determination of the $s, \tau_{\text {ref, }}$ and $n_{\text {out }}$ values is reduced to the following logical scheme:

$$
S_{H} \cdot A_{1} \stackrel{5}{\downarrow} A_{2} A_{3} P_{1} \stackrel{1}{\uparrow} A_{4} \stackrel{2}{\uparrow} \stackrel{1}{\downarrow} A_{5} \stackrel{2,3}{\downarrow} A_{6} P_{2} \stackrel{3}{\uparrow} A_{7} \stackrel{4}{\downarrow} A_{8} A_{9} P_{3} \stackrel{4}{\uparrow} A_{10} A_{11} A_{12} P_{4} \stackrel{5}{\uparrow} A_{13} S_{K} .
$$

In (38), the following designations are used:

- $S_{H}$ and $S_{K}$ are the start and end operators, respectively;

$-A_{1}$ is setting the number of $n_{\text {ref }}$ implementations and the duration of the reference pulse $t_{I M P}(i) \in t_{I M P}$;

$-A_{2}$ is the measurement of the current value $f(i)$;

$-A_{3}$ is the registration of the current values $t(i)$ and $f(i)$ as the initial conditions of the transition, that is, $t_{h}(i) \rightarrow t_{h}$, $f(i) \rightarrow f_{h}(i)$;

$-A_{4}$ and $A_{5}$ is setting " 1 " in the "Decrease" and "Increase" channels, respectively;

$-A_{6}$ is the formation of $t_{I M P}$ influences on the GM output;

$-A_{7}$ is the registration of the current value $f(i)$ as $f_{P}(i)$, that is, determining the moment of removal of the influence $t_{I M P}(i)$;

$-A_{8}$ is the measurement of $f_{i}(i)$ and $f_{i+1}(i)$ at interval $\tau=t_{i+1}-t_{i}$;

- $A_{9}$ - the calculation of $\Delta f(\tau)=\left|f_{i+1}(i)-f_{\mathrm{i}}(i)\right|$;

$-A_{10}$ is the registration of $t(i)$ and $f(i), \mathrm{t}(\mathrm{i})$ as the final conditions of transition, that is, $t_{K}(i)$ and $f_{K}(i)$;

$-A_{11}$ - the calculation of $s(i), t_{p}(i), \tau_{\text {ref }}(i)$ and $n_{\text {out }}(i)$ according to the formulae (33), (34), (36), and (38), respectively;

$-A_{12}$ is the decrease of the number by unity and recording $n_{\text {ref }}=n-1$;

$-A_{13}$ - the calculation of $s(M), \tau_{\text {ref }}(M), n_{\text {out }}(M)$ values for $t_{N P}(i)$ by formulae (33), (35), (37);

$-P_{1}, P_{2}, P_{3}, P_{4}$ are the conditions for the implementation of ratios $f(i)>f_{\text {nom }}, t_{N P}(i)-1=0, \Delta f(\tau)<\Delta \mathrm{f}(\tau)_{\text {dop }}$ and $n=0$, respectively;

$-\omega$ is the unconditional transition.

Thus, we have theoretically and practically proven the effectiveness of the use of the proposed technique to solve the problem of optimal frequency adjustment control at system parameters that vary during operation.

\section{Determining the moment of enabling generator assemblies at the deterministic and stochastic statement of the synchronization task}

Based on (7) to (9), we can conclude that determining the optimal moment of the coincidence of trajectories of synchronized objects is associated with solving a set of tasks. First, determining the mutual location in the vector space of the same-name voltage phases of the synchronized objects. Second, calculating the timeout of synchronization optimum, that is, the moment of geometric coincidence of the named vectors, the formation of an advance pulse to enable the synchronized object's generator assembly. In addition, the defined statement (11) and the determined progress in solving synchronization tasks (12) have made it possible to start solving the totality separated from them.

According to the deterministic statement (4), the mutual location in the vector space of the same-name voltage phases of synchronized objects is described by expression (6). Taking into consideration the fact that at the time of synchronization optimum the equality ${\overline{r_{S}}}^{(0)}=0$, holds, one can record:

$$
\Delta \bar{r}_{S Y}^{(k)}=\bar{r}_{O S}^{(k)}-\bar{r}_{S}^{(k)}
$$

where

$$
\begin{aligned}
& \Delta \bar{r}_{S}^{(k)}=\bar{r}_{S}^{(k)}-\bar{r}_{S}^{(0)}=\left[t^{(k)}-t^{(0)}\right] \frac{d \omega_{S}^{(k)}}{d t} \cong \\
& \cong\left|\Delta u_{S}^{(k)}\right|=\bigcup_{O S} \bigcup_{S Y} \sin \frac{\omega_{S}}{2}\left[t^{(k)}-t^{(0)}\right]
\end{aligned}
$$

- is the vector that determines changes in the value of the voltage that bends over the time $\left[t^{(k)}-t^{(0)}\right]$. Since the vector $\bar{r}_{S}^{(k)}=\bar{r}_{S Y}^{(k)} \cdot \bar{r}_{O S}^{(k)} \cdot \sin \frac{\alpha^{(k)}}{2}$, as well as taking into consid eration that $\bar{r}_{S}^{(0)}=0$ and $\frac{d \omega_{S}}{d t}=0$ (the mode of "freezing" of the synchronized object), then, after the task is removed, the object "falls out" of the synchronization process, that is:

$$
\bar{r}_{S Y}^{(k)} \bar{r}_{O S}^{(k)} \cdot \sin \frac{\alpha^{(k)}}{2} \cong \bigcup_{S Y} \bigcup_{O S} \sin \frac{\omega_{S}}{2}\left[t^{(k)}-t^{(0)}\right],
$$

hence

$$
\alpha^{(k)} \cong \omega_{S}\left[t^{(k)}-t^{(0)}\right] \text { or }\left[t^{(k)}-t^{(0)}\right] \cong t_{a v t}=\frac{\alpha^{(k)}}{\omega_{S}} .
$$

Thus, the dependence of the value $t_{w}$ on the current values of $\omega_{S}$ and $\alpha$ is determined, which makes it possible to start developing the corresponding mathematical support for SAEPP MP-system, taking into consideration its capabilities and the accepted techniques of measurement. The basis of our technique for measuring the values $\omega_{S}$ and $\alpha$ is the principle of counting the periods of time intervals of the sinusoidal voltages USY, UOS over the time interval that characterizes the difference in phases.

An MP system contains the necessary digital signal shapers that reflect the sequences of periods of voltages of the synchronized objects $\left(T_{S Y}=\frac{1}{f_{S Y}}\right)$ and $\left(T_{O S}=\frac{1}{f_{O S}}\right)$ (Fig. 3), as well as a logical device for adding digital signals, which determines the angle $\alpha$ at the TS time interval and fully corresponds to the curve that bends the specified period:

$$
u_{S}=2 U_{m} \sin \frac{\omega_{S}}{2} t \text { at } U_{S Y}=U_{O S}=U_{m} .
$$

The main difficulties that have to be encountered in the development of mathematical support for MP devices for 
managing GS synchronization are obtaining an estimation expression for the $t_{w}$ time value from the beginning of the measurement $(I)$ of angle $\alpha$ to the optimum point $0_{2}$. This is due to the fact that during $\mathrm{T}_{S}$ the values of angles $\alpha$ in symmetrical sections $0_{1} M$ and $M 0_{2}$ coincide pairwise. Therefore, the use of dependence (39) to determine the time $t_{w}$ will be true only in section $\mathrm{MO}_{2}$ :

$$
t_{w}=\frac{\alpha}{\omega_{S}}=\frac{\alpha}{2 \pi} \cdot T_{S} .
$$

Assessing the ways to solve this task, we shall dwell on the idea of measuring two consecutive values of angle $\alpha$ and then assessing the sign of their difference. In this case, one can establish the following: if $\alpha_{1}<\alpha_{2}$, the process of measuring $\alpha$ is performed on section $0_{1} M$, at $\alpha_{1}>\alpha_{2}-\operatorname{section} M 0_{2}$.

However, in cases of complete imposition of time intervals $\tau_{i}$ and $\tau_{j}$, the marked pattern may be violated, which would lead to an error in determining the sign. For a given case, the $\alpha$ values are considered minimal ( $\alpha^{*}$, Fig. 3 ) and would always be less than $\Delta T=\left|T_{O S}-T_{S Y}\right|$. Therefore, to exclude possible errors, the measured values must be checked against $\alpha>\Delta T$.

Thus, one can write the following expressions to determine the desired parameter $t_{w}$ :

$$
\begin{aligned}
& t_{w}=\left(1-\frac{\alpha}{2 \pi}\right) T_{S}, \text { if } \alpha_{1}<\alpha_{2} ; \\
& t_{w}=\frac{\alpha}{2 \pi} \cdot T_{S}, \text { if } \alpha_{1}>\alpha_{2},
\end{aligned}
$$

or, given $T_{S}=\frac{T_{S Y} T_{O S}}{\left|T_{S Y}-T_{O S}\right|}, \quad T_{O S}=2 \tau(j)$ and $T_{S Y}=2 \tau_{S Y}$, we shall receive in the final form the expressions necessary to solve the set task by using the proposed method:

$$
t_{w}=\left(2-\frac{\alpha}{\pi}\right) \cdot \frac{\tau_{O S} \cdot \tau_{S Y}}{\tau_{O S}-\tau_{S Y}}, \text { if } \alpha_{1}<\alpha_{2},
$$

$$
t_{w}=\frac{\alpha}{\pi} \cdot \frac{\tau_{O S} \cdot \tau_{S Y}}{\tau_{O S}-\tau_{S Y}}, \text { if } \alpha_{1}>\alpha_{2} .
$$

Under the proposed approach to determining $t_{\mathfrak{w}}$, the value of delay in time $\tau_{A D V}$ to trigger the advance pulse for enabling the generator assembly can be calculated from the following formula:

$$
\tau_{A D V}=t_{w}-t_{a v t},
$$

where $t_{\text {avt }}$ is the average time of actuating the elements in a GS enabling channel.

Expression (42) shows the accuracy of connecting a synchronized object to an object of synchronism depends not only on the accuracy of the measurement of values $\alpha$ and $\omega_{S}$ and the calculation of $t_{w}$ but also on the probability of the current value of the $t_{\text {avt }}$ value.

Based on (10), one can write for the probability of not exceeding the service time of a given value: $t_{a v t}(i)=\tau_{A B}(i), \tau_{p 1}(i)$, $\tau_{p 2}(i), \tau_{c s}(i)$. Moreover, $\tau_{A B}, \tau_{p 1}, \tau_{p 2}$ and $\tau_{c s}$ is the natural time of operation of the main elements in an GS enabling channel, respectively: control system $\tau_{C S}$, galvanic solution relay $\tau_{p 1}$, intermediate relay $\tau_{p 2}$, and a generator circuit breaker $\tau_{A B}$.

In fact, during the SAEPP operation, their time indicators $\tau_{A B}, \tau_{p 1}, \tau_{p 2}, \tau_{C S}$ change, which leads to significant changes in $t_{\text {avt }}$ and, as a result, to errors in the calculation of $t_{a v t}$. Since the probability of the last $k$-enabling out of $n$ preceding the calculations of the value $t_{\text {avt }}(n+1)$ are more likely, one can use the following formula to determine the latter

$$
\begin{aligned}
& t_{a v t}(n+1)= \\
& =\frac{\sum_{i=n-k}^{n}\left(\tau_{A B}(i)+\tau_{p 1}(i)+\tau_{C S 2}(i)+\tau(i)\right)}{k} .
\end{aligned}
$$

Thus, it can be stated that (43) underlies determining the optimal moment for enabling the generator assembly of the synchronized object under the conditions of the deterministic setting of tasks for managing the process of GS synchronization.

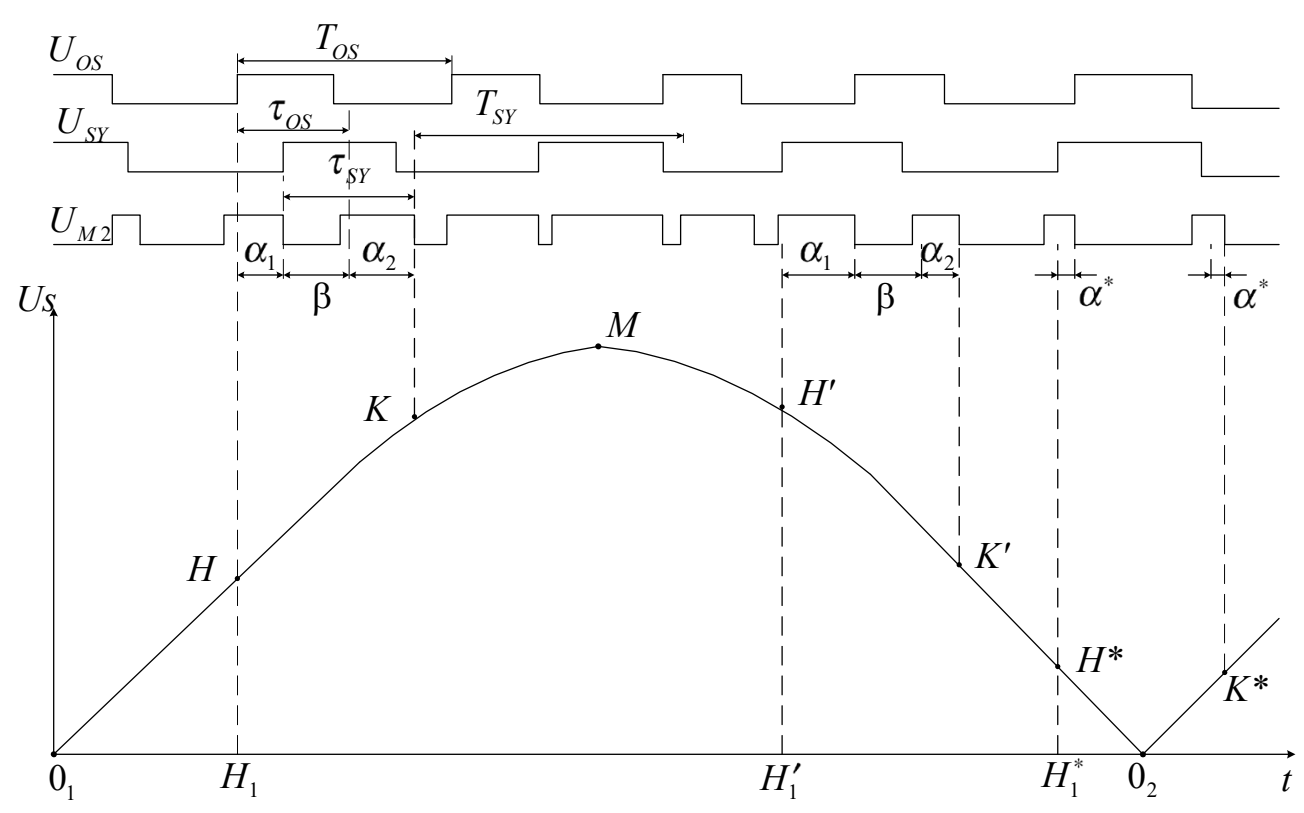

Fig. 3. Illustration of the technique for determining the synchronization parameters 
The stochastic approach to solving these tasks is predetermined by that in real conditions the SAEPP with SG is exposed to stochastic disturbances, both from the propeller shaft and from the ship's network. In this case, external disturbing influences can cause such significant changes, not taking into consideration which, when determining the optimal moment of switching on the generator of the synchronized object, can lead to unsuccessful synchronization. In this regard, there is a need to predict changes in $T_{C}$ and $\mathrm{T}_{S G}$ in order to correct the value of $t_{w}$, check the conditions for stability, and form control influences.

To solve this problem, it is necessary to have a certain set of parameters that characterize a random process statistically related to the observed one. This set includes:

$$
\begin{aligned}
& T_{a v}=\frac{\sum_{i=1}^{n} T_{i}}{n} ; A_{a v}=\frac{\sum_{i=1}^{n}\left|A_{i}\right|}{n} ; \\
& \frac{\Delta x}{\Delta t} a v=\frac{\sum_{i=1}^{m}\left|x_{i}-x_{i-1}\right|}{m \cdot t_{d u r}},
\end{aligned}
$$

where $T_{i}$ is the $i$-th period of the random process $x(t)$, and $i$ is the number of periods during the implementation of $t ; A_{i}=\left|x_{i}-x_{i-1}\right|$ is the deviation of the observed value $x\left(T_{C}\right.$ or $\left.T_{S G}\right)$ from its rated value $x_{\text {nom }}$ at the points of an extremum $x_{i}^{+}$and $x_{i}^{-} ; \frac{\Delta x}{\Delta t} a v$ is the average rate of change in $x$ in $m$ steps of registration; $t_{d u r}$ is the duration of the registration step.

The selected value of $t_{d u r}$ will largely determine such contradictory indicators of measuring process parameters as accuracy and machine time cost. Therefore, given that $\{T, A, \Delta x / \Delta t\}$ under real conditions vary in wide ranges, the choice of $t_{d u r}$ should be adaptive. Since the variables $\{T, A, \Delta x / \Delta t\}$ are represented discretely in an MP system, then we assume that $t_{d u r}=f(T, A, \Delta x / \Delta t)$ is a lattice function with a variable discrete interval specified by recurrent ratios or tables.

If we assume that the cyclicity of access to the measurement process is set and characterized by a certain period $T_{0}$, and:

$$
\mathrm{T}_{0}=t_{\text {meas }}+t_{\Pi} ; t_{\text {meas }}=N \cdot t_{p} ; t_{p}=K \cdot t_{d u r},
$$

and $K, N$, and $t_{\text {meas }}$ are, respectively, the number of registration points during time $t_{p}$, the number of implementations during measurement time $t_{\text {meas }}$, and the duration of the pause between measurements.

By setting the optimal value $t_{d u r}$ in this way, we introduce it in the measurement algorithm.

That makes it possible to conclude that it is advisable to search for a correction to manage the synchronization process and choose a rational technique of calculation. The conclusion of expediency can be drawn on the basis of the analysis of the ratios of values that characterize the observed process $\left\{T_{C}, T_{S G}, T S\right\}$ and the statistically related random process $\left\{T_{a v}, A_{a v}, \Delta x / \Delta t\right\}$.

\section{Discussion of results of synthesizing a synchronization process control system}

If the obtained results are generalized and formalized taking into consideration the chosen strategy, then the structure of dual control over a synchronization process would take the form shown in Fig. 4.

In this regard, solving the set task using the theory of dual controlacquires the properties of the established correspondence between the model of transition $\omega_{S Y}(H) \stackrel{t_{M P}}{\longrightarrow} \omega_{S Y}\left(K_{M}\right)$ and the values of $s(M), \tau_{\text {ref }}(M), n_{\text {out }}(M)$.

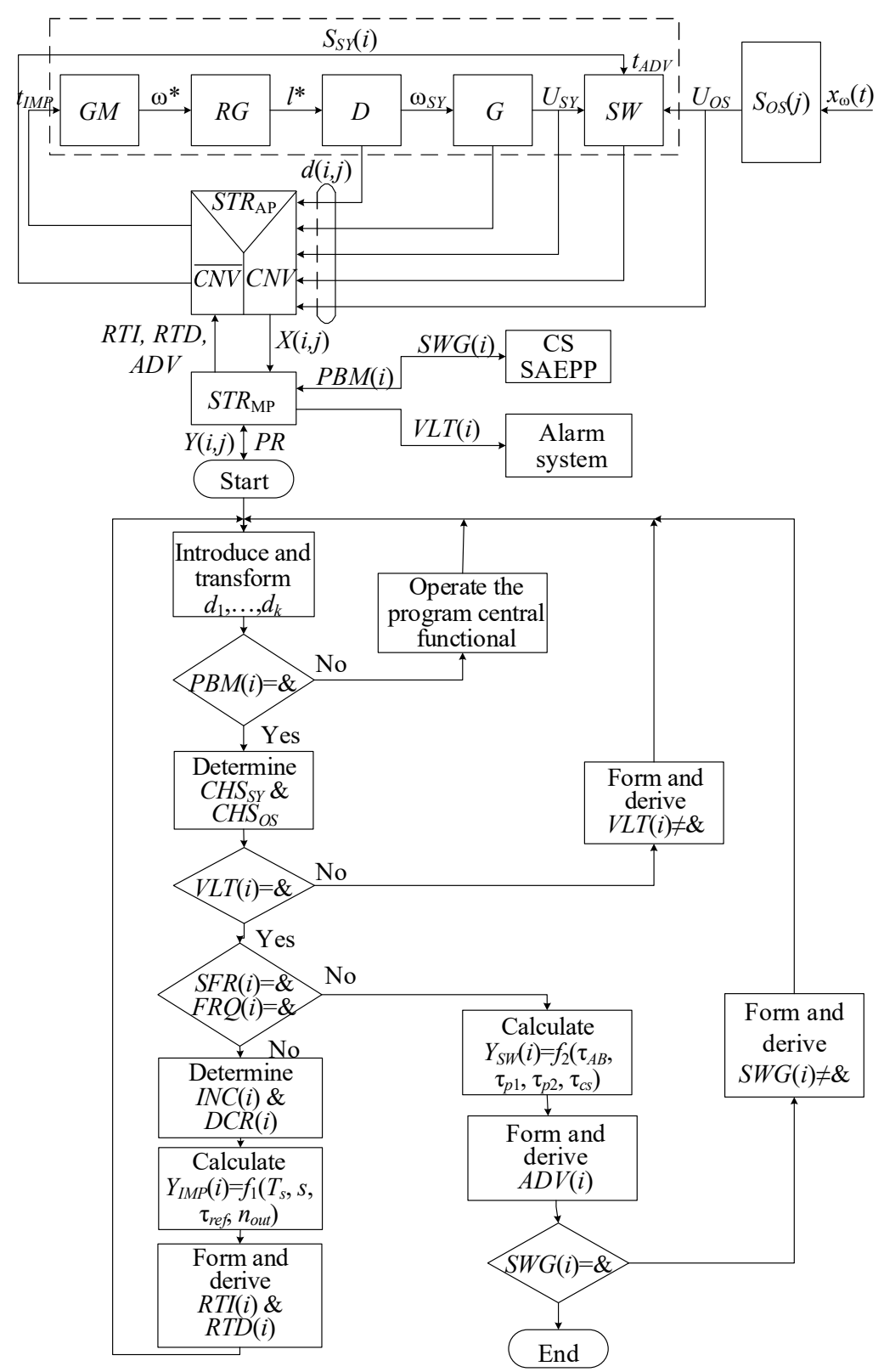

Fig. 4. Generalized structure of dual control over a GS synchronization process

Property 1. The ratios $\gamma_{m} / \gamma_{n}=$ const, $\gamma_{0} / \gamma_{n}=$ const that characterize the transition process are proportional to the adjustment coefficients $K_{n}=\operatorname{ctg} \gamma_{m} / \operatorname{ctg} \gamma_{n}$ and $K_{0}=\operatorname{ctg} \gamma_{0} / \operatorname{ctg} \gamma_{n}$.

Property 2. There is a point $M\left(\omega_{M}, \mathrm{t}_{M}\right)$, the one that

$\omega_{M}=\omega_{S Y}(H)+1 / 2 \omega_{S}(H), t_{M}=t_{H}+\frac{1}{2} t_{p}$. 
Taking into consideration "Property 1", according to $\gamma_{n} \cong s(M), \quad t_{p=} \omega_{s}(H) \cdot \operatorname{ctg} \gamma_{M}$ and (46), we obtain

$$
t_{p}=\frac{K_{n} \omega_{s}(H)}{s(M)} .
$$

The defined dependences $\Delta f=h_{1}\left(t_{I M P}\right)$ and $t_{p}=h_{2}(\Delta f)$ confirm the practical feasibility of the given algorithm (Fig. 4) for ship MP-systems of control when automatically determining $s, \tau_{r e f}$ and $n_{\text {out }}$ and using a technique to identify the values of the transition process $s$ and $t_{p} \mathrm{~s}$ according to the characteristics $\Delta f=h_{1}\left(t_{I M P}\right)$ and $t_{p}=h_{2}(\Delta f)$ (Fig. 5). The initial and final values of frequency $f_{h}$ and $f_{k}$ and the time $t_{h}$ and $t_{k}$ of the corresponding transition process are recorded from the chain $G M \rightarrow R G \rightarrow G S$ in a set of reference values $t_{I M P}^{S T}=\{1,2,3,4\}$ in the memory of an MP system.

The transition model

$$
\omega_{S Y}(H) \stackrel{t_{M P}}{\longrightarrow} \omega_{S Y}\left(K_{M}\right)
$$

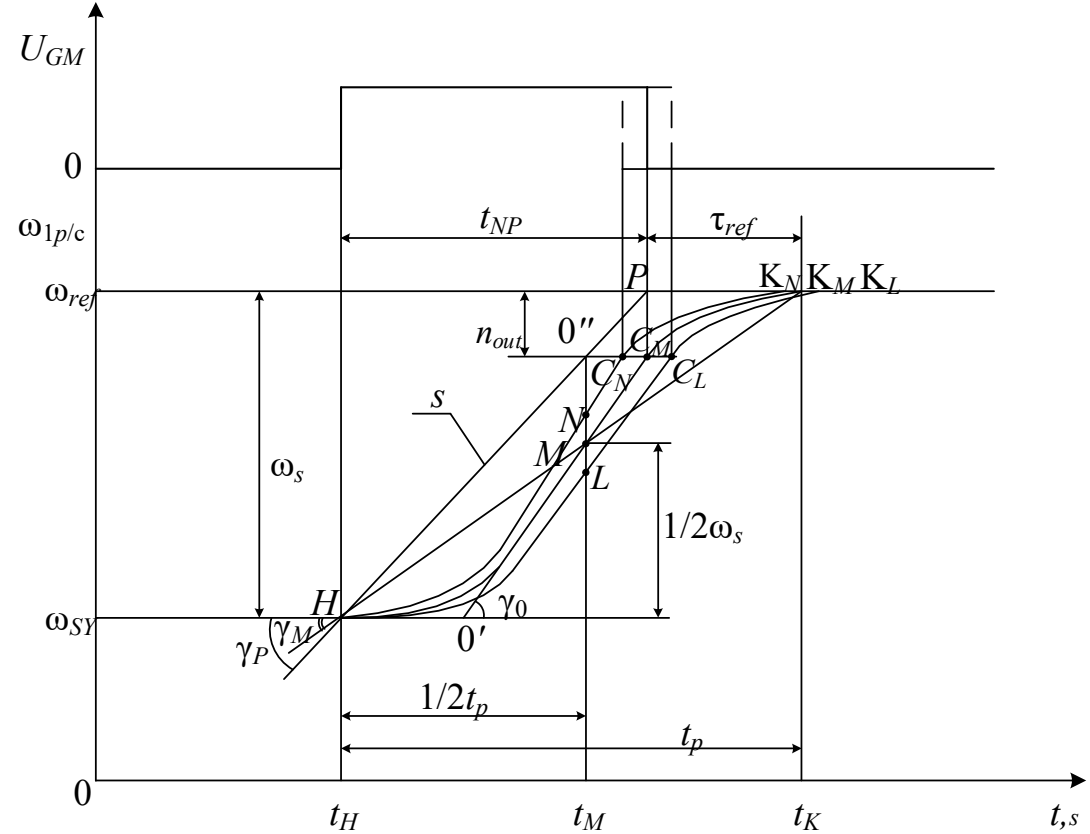

Fig. 5. Illustration of the method of dual control over frequency adjustment is represented as an aperiodic process (Fig. 5, HMKM curve) and is one that corresponds to a given model and determines the characteristics $B(M)=\left(s(M), \tau_{r e f}(M)\right.$ and $\left.n_{\text {out }}(M)\right)$. These values characterize the transition process in the $G M \rightarrow R G \rightarrow G S$ chain, which allows using them as initial data in the synthesis of an algorithm for the optimal frequency adjustment control and in assessing the dynamic properties of the chain's elements.

The use of the algorithm of dual control over synchronization (Fig. 4) applying the procedure for determining the moment of switching on generator assemblies has made it possible to adjust the frequency (Fig. 6, $a$ ) and time characteristics (Fig. 6, $b$ ) under the stochastic and deterministic statement of synchronization tasks.

The proposed approach has made it possible to investigate the process of synchronization when an object of synchronism moves along the predefined trajectory with stochastic fluctuations regarding its task under the influence of external factors.

The method of dual control over frequency adjustment and for determining the frequency adjustment time characteristics (Fig. 6) has made it possible to achieve stable parallel operation of the diesel generators even under the conditions of perturbations under load. Experimental operational parameters of generator No. 2 under the conditions of perturbations under load are shown in Fig. 7.

The experiment on the synchronization of the diesel generators under the conditions of scholastic perturbations was carried out using the variable load on SAEPP. The load change was carried out by a random law by controlling the frequency and torque of AC motors used as SAEPP consumers.

The process of the failed synchronization when using standard algorithms for managing the synchronizer, implemented on DEIF PPM-3 controllers, is shown in Fig. 8. One can see that immediately after switching on generator No. 2 in parallel, the system becomes unstable (load fluctuations: full - white chart, active - green chart) and, after a short period of time, the protection unit disconnects generator No. 1 from MSB buses.
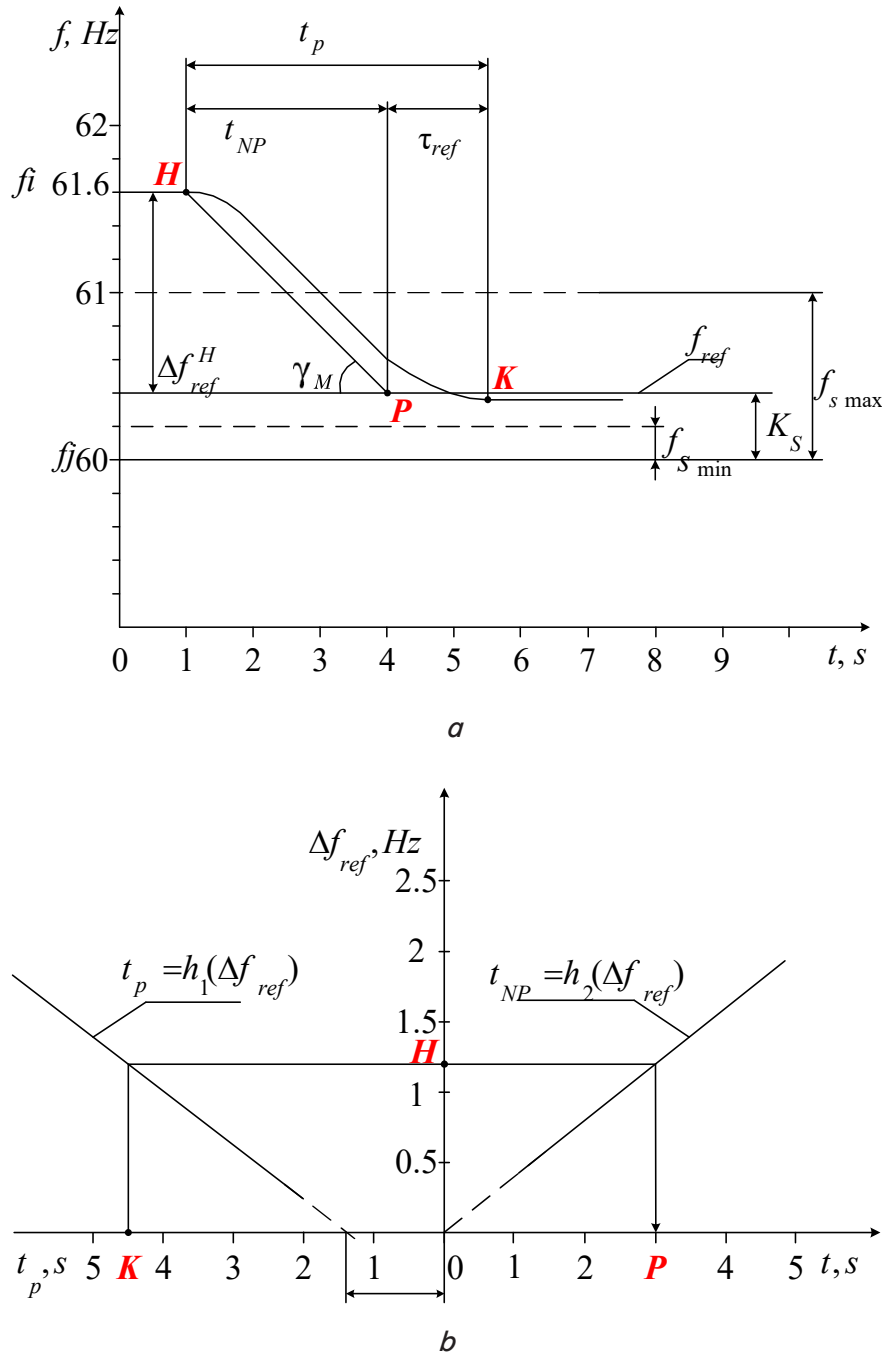

Fig. 6. Illustration of the process: $a-$ frequency adjustment; $b$ - determining time characteristics 


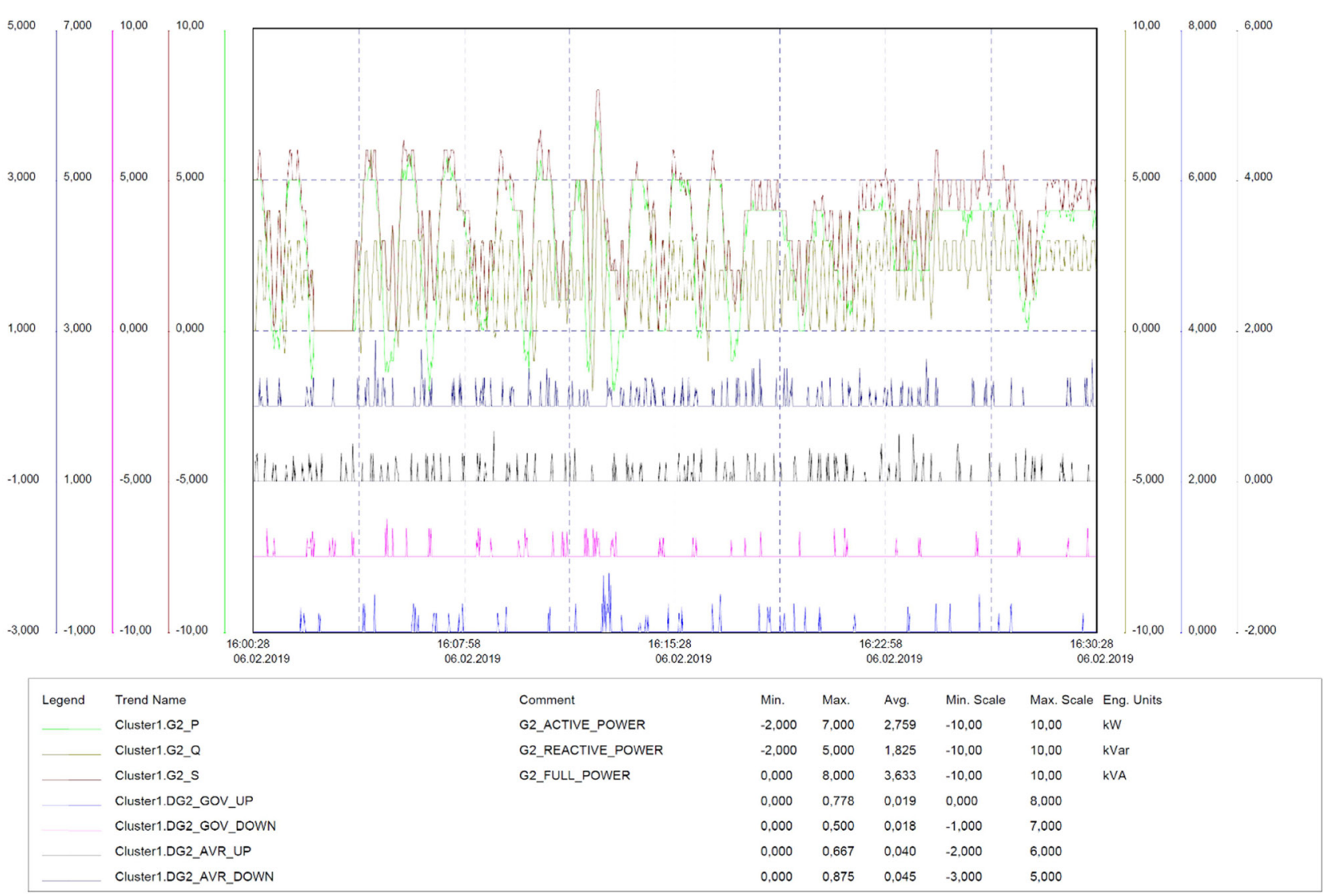

Fig. 7. Experimental processes of change in the parameters of generator No. 2 under the conditions of perturbations under load

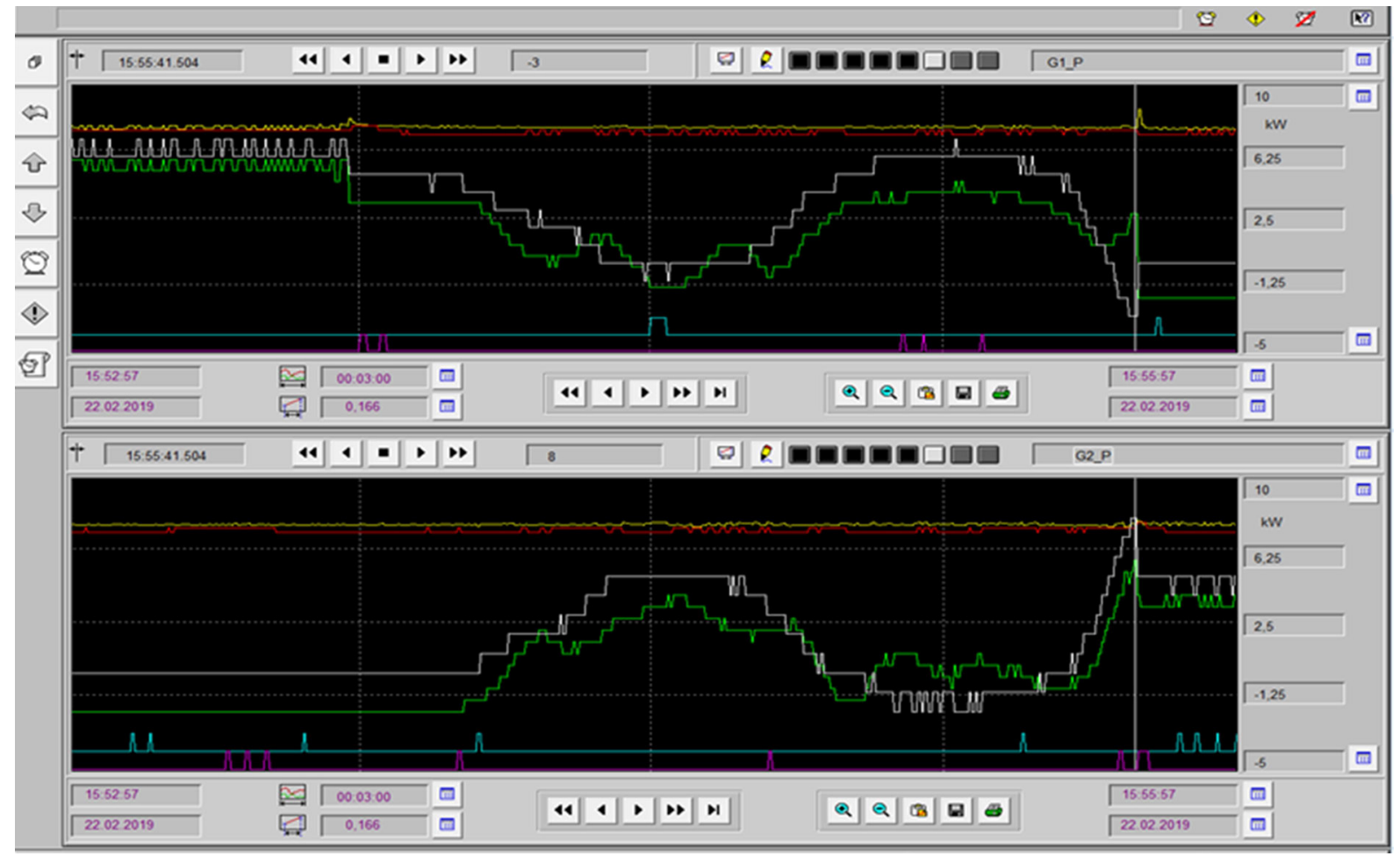

Fig. 8. Process of failed synchronization when using standard control algorithms

The use of advanced algorithms of dual control over synchronization (applying the proposed procedure for determining the moment of enabling GS) under the stochastic setting of the syn- chronization task has made it possible to achieve effective successful synchronization and stable parallel operation of the diesel generators even under the conditions of perturbations (Fig. 9). 


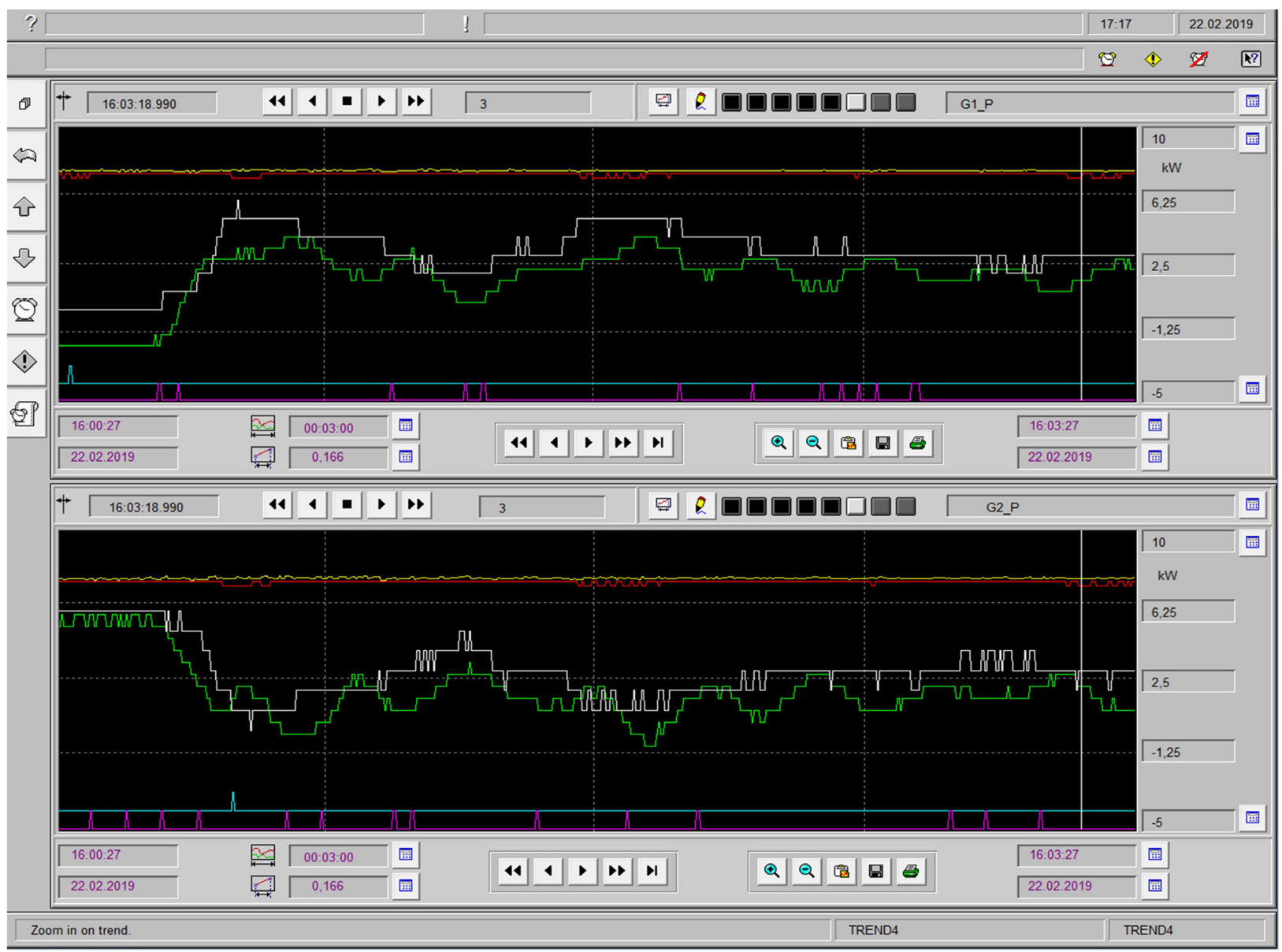

Fig. 9. Process of successful synchronization when applying dual control algorithms

Further improvement of the proposed stochastic approach could involve defining a general optimization criterion for the dual control over synchronization process. And advancing, in turn, the universal structure of synchronization process control - through the practical testing of synchronization control techniques under real ship conditions.

\section{Conclusions}

1. The set task of GS synchronization was solved by constructing process models, their databases, and by determining the main possible configurations of control systems. Based on the application of resultant functions, the models of the main converters were described on the basis of the use of the principle of relative movement of objects. Using ship GSs as an example, it has been proven that the improved functioning of the elements in controlling complex STS and $\mathrm{C}$ can be achieved when using the integrated optimization criteria, as well as dual control principles.

2. The stages in solving frequency adjustment synchronizai tion tasks are arranged in the following hierarchical sequence:

- formalize the set of design tasks in accordance with technical-ergatic and ergonomic requirements for a SAEPP MP control system;

- synthesize the initial logical-mathematical model of the designed MP-system, description the set of characteristics, system quality functions;
- select research methods and analyze the logical-matht ematical model in accordance with the set task of synchronization;

- devise ways to solve the problems of control and syny thesis of the system of functional algorithms (determining the composition and number of operations, operands and constants, the valid calculation time, the volume of input parameters and maximum errors of their measurement, the nature of data exchange with external devices);

- test and debug in the process of joint operation of the hardware and software parts.

3. Determining the optimization criteria for the dual control over the GS synchronization process has made it possible to implement the law of control, which satisfied the condition for an extremum, which is an indicator of the feasibility of the set goal and takes into consideration the limitations for control influences and changes in the characteristics of synchronization objects. Our system in the class of adaptive control systems was designed owing to the appropriate decomposition of system elements by splitting a synchronization problem into a performance task and a control task under the required conditions.

4. Our examples of the processes of failed synchronizai tion when using standard algorithms of synchronizer control and the processes of successful GS synchronization while applying the proposed algorithms of dual control over the synchronizer have confirmed the reliability of the main scientific results reported here. 


\section{References}

1. Boveri, A., Silvestro, F., Gualeni, P. (2016). Ship electrical load analysis and power generation optimisation to reduce operational costs. 2016 International Conference on Electrical Systems for Aircraft, Railway, Ship Propulsion and Road Vehicles \& International Transportation Electrification Conference (ESARS-ITEC). doi: https://doi.org/10.1109/esars-itec.2016.7841422

2. Shevchenko, V. A. (2018). Optimization of the process of automatic synchronization of ship diesel generators in the deterministic formulation of the problem. Automation of technological and business processes, 10 (4), 43-53. doi: https://doi.org/10.15673/ atbp.v10i4.1233

3. Pipchenko, A. N., Ponomarenko, V. V., Teplov, Yu. I., Shevchenko, V. A. (2019). Elektrooborudovanie, elektronnaya apparatura i sistemy upravleniya. Odessa: TES, 567.

4. Carri $(n, M ., Z$ Z rate-Mi〉ano, R., Milano, F. (2020). Impact of off-nominal frequency values on the generation scheduling of smallsize power systems. International Journal of Electrical Power \& Energy Systems, 122, 106174. doi: https://doi.org/10.1016/ j.ijepes.2020.106174

5. Ghaedi, A., Golshan, M. E. H. (2021). Modified WLS three-phase state estimation formulation for fault analysis considering measurement and parameter errors. Electric Power Systems Research, 190, 106854. doi: https://doi.org/10.1016/j.epsr.2020.106854

6. Xu, F., Yang, W., Li, H. (2020). Computation offloading algorithm for cloud robot based on improved game theory. Computers \& Electrical Engineering, 87, 106764. doi: https://doi.org/10.1016/j.compeleceng.2020.106764

7. Heinrich, B., Krause, F., Schiller, A. (2019). Automated planning of process models: The construction of parallel splits and synchronizations. Decision Support Systems, 125, 113096. doi: https://doi.org/10.1016/j.dss.2019.113096

8. Kumar, J., Kumpulainen, L., Kauhaniemi, K. (2019). Technical design aspects of harbour area grid for shore to ship power: State of the art and future solutions. International Journal of Electrical Power \& Energy Systems, 104, 840-852. doi: https:// doi.org/10.1016/j.ijepes.2018.07.051

9. Chen, H. (2020). Simulation Research on Ship Electric Propulsion Speed Regulation System Based on Variable Structure Control and FPGA. Microprocessors and Microsystems, 103588. doi: https://doi.org/10.1016/j.micpro.2020.103588

10. Aiello, G., Giallanza, A., Vacante, S., Fasoli, S., Mascarella, G. (2020). Propulsion Monitoring System for Digitized Ship Management: Preliminary Results from a Case Study. Procedia Manufacturing, 42, 16-23. doi: https://doi.org/10.1016/j.promfg.2020.02.018

11. Attia, A.-F., Sharaf, A. M. (2020). A robust FACTS based fuzzy control scheme for dynamic stabilization of generator station. Ain Shams Engineering Journal, 11 (3), 629-641. doi: https://doi.org/10.1016/j.asej.2019.11.004

12. Emam, S. E. A. (2004). Automaic digital synchronization. International Conference on Electrical, Electronic and Computer Engineering, 2004. ICEEC '04, 778-784. doi: https://doi.org/10.1109/iceec.2004.1374594

13. Guzzella, L., Onder, C. H. (2010). Introduction to Modeling and Control of Internal Combustion Engine Systems. Springer. doi: https:// doi.org/10.1007/978-3-642-10775-7

14. Mi, Y., Xu, Y., Lang, Z., Yang, X., Ge, X., Fu, Y., Jin, C. (2021). The frequency-voltage stability control for isolated wind-diesel hybrid power system. Electric Power Systems Research, 192, 106984. doi: https://doi.org/10.1016/j.epsr.2020.106984

15. Myrhorod, V., Hvozdeva, I., Budashko, V. (2020). Multi-parameter Diagnostic Model of the Technical Conditions Changes of Ship Diesel Generator Sets. 2020 IEEE Problems of Automated Electrodrive. Theory and Practice (PAEP). doi: https:// doi.org/10.1109/paep49887.2020.9240905

16. Sadeghian, Z., Akbari, E., Nematzadeh, H. (2021). A hybrid feature selection method based on information theory and binary butterfly optimization algorithm. Engineering Applications of Artificial Intelligence, 97, 104079. doi: https://doi.org/10.1016/ j.engappai.2020.104079

17. Shevchenko, V. A. (2018). Ships electrical power plant control system top level algorithm synthesis method and specifics. Visnyk Skhidnoukrainskoho natsionalnoho universytetu imeni Volodymyra Dalia, 6 (247), 165-174.

18. Boyko, A., Budashko, V., Yushkov, Y., Boyko, N. (2016). Synthesis and research of automatic balancing system of voltage converter fed induction motor currents. Eastern-European Journal of Enterprise Technologies, 1 (2 (79)), 22-34. doi: https:// doi.org/10.15587/1729-4061.2016.60544

19. Budashko, V., Shevchenko, V. (2018). Synthesis of the Management Strategy of the Ship Power Plant for the Combined Propulsion Complex. 2018 IEEE 5th International Conference on Methods and Systems of Navigation and Motion Control (MSNMC). doi: https://doi.org/10.1109/msnmc.2018.8576266

20. Budashko, V. (2020). Thrusters Physical Model Formalization With Regard to Situational and Identification Factors of Motion Modes. International Journal of Energy and Environment, 14, 5-8. doi: https://doi.org/10.46300/91012.2020.14.2

21. Karatas, B. C., Sarkar, M., J Channsson, H., Nielsen, A. H., S rensen, P. E. (2020). Voltage stability assessment accounting for currentlimited converters. Electric Power Systems Research, 189, 106772. doi: https://doi.org/10.1016/j.epsr.2020.106772

22. Kowalski, J., Krawczyk, B., Woźniak, M. (2017). Fault diagnosis of marine 4-stroke diesel engines using a one-vs-one extreme learning ensemble. Engineering Applications of Artificial Intelligence, 57, 134-141. doi: https://doi.org/10.1016/j.engappai.2016.10.015

23. Luo, T., Zhang, L., Zhang, C., Ma, J., Xu, Z., Sun, X., Zhao, S. (2018). Role of water as the co-solvent in eco-friendly processing oil extraction: Optimization from experimental data and theoretical approaches. Chemical Engineering Science, 183, $275-287$. doi: https://doi.org/10.1016/j.ces.2018.03.015 
24. Motienko, A. (2020). Integration of information and communication system for public health data collection and intelligent transportation system in large city. Transportation Research Procedia, 50, 466-472. doi: https://doi.org/10.1016/j.trpro.2020.10.055

25. Nuchturee, C., Li, T., Xia, H. (2020). Energy efficiency of integrated electric propulsion for ships - A review. Renewable and Sustainable Energy Reviews, 134, 110145. doi: https://doi.org/10.1016/j.rser.2020.110145

26. Pakshina, N. A., Pravdina, M. V., Koposov, A. S., Pakshin, P. V. (2017). Team Public Testing in Classroom Studies on Automatic Control Theory. IFAC-PapersOnLine, 50 (1), 13468-13473. doi: https://doi.org/10.1016/j.ifacol.2017.08.2318

27. Pipchenko, A. D., Shevchenko, V. A. (2018). Vessel heading robust automatic controller for varying conditions. Marine Intellectual Technologies, 4 (4 (42)), 208-214.

28. Gaysarov, R. V. (2005). Rezhimy raboty elektrooborudovaniya elektricheskih stantsiy i podstantsiy: Chast' 1. Rezhimy raboty sinhronnyh generatorov i kompensatorov. Konspekt lektsiy. Chelyabinsk: Izd-vo YuUrGU, 42.

29. Dorogan', O. I. (2013). Mikroprotsessornye sredstva upravleniya parallel'noy rabotoy dizel'-generatornyh agregatov. Mater. Vseukr. nauk.-tekhn. konf. z mizhnarodnoiu uchastiu. Mykolaiv, 3-7.

30. Zaharchenko, V. N., Shevchenko, V. A. (2015). Reshenie zadach upravleniya sudovoy elektroenergeticheskoy ustanovkoy pri izmenenii nagruzki. Sudovye energeticheskie ustanovki, 36, 74-82.

31. Kutyashova, A. Yu. (2011). Usovershenstvovanie sistem sbora i otobrazheniya informatsii na energoobektah. Sbornik dokl. 3-y vseross. konf. «Rekonstruktsiya energetiki - 2011». Moscow: OOO «Inteheko», 81-85.

32. Pavlenko, M. A., Rudenko, V. N., Berdnik, P. G., Danyuk, Y. V. (2010). Systems of support of making a decision and task of their ergonometric planning. Military Technical Collection, 3, 3-7. doi: https://doi.org/10.33577/2312-4458.3.2010.3-7

33. Pavlov, G. M., Merkur'ev, G. V. (2015). Avtomatika energosistem. NOU "Tsentr podgotovki kadrov energosistem". Sankt-Peterburg. Available at: https://rza.org.ua/down/open/Avtomatika-energosistem--G-M--Pavlov--G-V--Merkurev.html

34. Dorohan', O. I. (2013). Pat. No. 82745 UA. Functional structure of logical-dynamic synchronization process of generator and network. No. u201300569; declareted: 17.01.2013; published: 12.08.2013.

35. Riabenkyi, V. M., Ushkarenko, O. O., Dorohan, O. I., Babak, V. I. (2013). Pat. No. 82749 UA. Method for conversion of analogue generator signal in logical-dynamic process of synchronization thereof with network. No. u201300575; declareted: 17.01.2013; published: 12.08 .2013 .

36. Savenko, O. Ye. (2013). Optimization of the ship's power plant to improve the quality of electricity. Visnyk of Vinnytsia Polytechnical Institute, 6, 74-78.

37. Savenko, O. Ye. (2011). Theoretical and experimental study of the multigenerating ship power system functioning. Visnyk of Vinnytsia Polytechnical Institute, 3, 58-62.

38. Tykhonov, I. V., Davydov, V. S., Kucheruk, S. M., Bohomia, V. I. (2013). Osnovy teoriyi pokhybok vymiriuvan. Kyiv, 66. 